\title{
A Robust Recursive Filter for Nonlinear Systems with Correlated Noises, Packet Losses, and Multiplicative Noises
}

\author{
Hua-Ming Qian, ${ }^{1}$ Wei Huang, ${ }^{1}$ Biao Liu, ${ }^{2}$ and Chen Shen ${ }^{1}$ \\ ${ }^{1}$ College of Automation, Harbin Engineering University, Harbin 150001, China \\ ${ }^{2}$ Dongguan Techtotop Microelectronics Co., Ltd., Chengdu R \& D Branch, Chengdu 610041, China \\ Correspondence should be addressed to Wei Huang; huangwei2393@163.com
}

Received 2 November 2013; Revised 16 January 2014; Accepted 24 January 2014; Published 31 March 2014

Academic Editor: Xiaojie Su

Copyright (c) 2014 Hua-Ming Qian et al. This is an open access article distributed under the Creative Commons Attribution License, which permits unrestricted use, distribution, and reproduction in any medium, provided the original work is properly cited.

\begin{abstract}
A robust filtering problem is formulated and investigated for a class of nonlinear systems with correlated noises, packet losses, and multiplicative noises. The packet losses are assumed to be independent Bernoulli random variables. The multiplicative noises are described as random variables with bounded variance. Different from the traditional robust filter based on the assumption that the process noises are uncorrelated with the measurement noises, the objective of the addressed robust filtering problem is to design a recursive filter such that, for packet losses and multiplicative noises, the state prediction and filtering covariance matrices have the optimized upper bounds in the case that there are correlated process and measurement noises. Two examples are used to illustrate the effectiveness of the proposed filter.
\end{abstract}

\section{Introduction}

In recent years, the state estimation theory has received extensive attention in many fields of application, such as attitude estimation [1], target tracking [2], signal processing [3], and integrated navigation [4]. State estimation refers to a methodology that is used for estimating the state of a time-varying system through noisy measurements, which are different from other methods [5-9]. So far, various kinds of filtering algorithms for state estimation have been presented, for example, Kalman filter [10], extended Kalman filter (EKF) [11], unscented Kalman filter (UKF) [12], and so forth. As is well known, among those filters, Kalman filter is an optimal solution based on the minimum mean square error rule for linear systems and EKF is an effective way for softly nonlinear system to estimate the state by using linearization techniques. Although EKF is a popular estimating algorithm in engineering practice, its use must satisfy the following two assumptions: (1) the system model should be accurate and (2) the additive noises should be Gaussian and uncorrelated. Otherwise, the performance of EKF can be degraded severely, even unstable. Unfortunately, in real world, the model uncertainty is an unavoidable and crucial problem for nonlinear systems. Therefore it is required to develop a more general filtering algorithm. To this end, the robust filtering technique has been developed to reduce the unfavorable effect of model uncertainties by establishing an appropriate uncertain model in consideration of uncertainties. Up to now, a lot of literatures on the robust filtering problem with model uncertainties have been published, such as the $H_{\infty}$ filter [13-16], set-valued nonlinear filter [17, 18], mixed $\mathrm{H}_{2} / \mathrm{H}_{\infty}$ filter $[19,20]$, and robust extended Kalman filter design [21, 22]. In these reports, the robust recursive filter design has been investigated to be available for handling the nonlinear filtering problem with model uncertainties. For instance, a discrete-time robust extended Kalman filter has been presented for uncertain systems with sum quadratic constraints in [21]. Due to the influence of the misalignments of star sensors, by considering the model uncertainties, a nonlinear robust filter for satellite attitude determination is developed and verified in [22].

In literature mentioned above, however, only additive noises are considered for nonlinear systems. Actually, another important noise called multiplicative noise is often encountered in many engineering systems, such as attitude estimation systems and airborne synthetic aperture radar systems. It 
is coupled with the state and has an unknown noise variance, which results in a negative impact on the state estimation. Hence, the multiplicative noise is usually viewed as a model uncertainty. Currently, the nonlinear robust filtering problem with multiplicative noises has been much less researched. In $[23,24]$, by utilizing linear matrix inequality approach, a robust Kalman filter is derived for linear systems. Different from them, another robust Kalman filter is proposed for linear systems by finding two Riccati differential equations and determining the filter parameters in [25]. Then, [26] extends the work to nonlinear systems. Apart from multiplicative noises, signal transmissions in the sensor networks are often unreliable. For example, sudden sensor failure, random communication delays, and packet losses appear in the practical system frequently [27-31]. All these lead to the measurement mode uncertainty. Accordingly, the filtering problem with packet losses has stirred considerable research attention and many research results have been published recently; see, for example [32-35]. In most literatures, the packet loss is described as a random variable in the distribution of Bernoulli, which may not be available because of the existence of the different transmission process in multiple sensors. In [36, 37], a diagonal matrix composed of Bernoulli random variables is introduced to the measurement equation, which means that individual sensor might have different missing rates. Meanwhile, it is not difficult to find that the most existing filtering researches concerning packet losses are subject to linear systems. However, as we all know, nonlinearity is inevitable in almost all engineering applications, which will directly degrade the quality of the filtering performance. For this purpose, a quantized recursive filtering is presented for a class of nonlinear systems with missing measurements, multiplicative noises, and quantization effects in [37]. Though missing measurements and multiplicative noises are taken into consideration at the same time, this work endures the limitation that the measurement equation must be linear, which makes that the algorithm in [37] cannot be extended to solve the general nonlinear filtering problems in the case that the process and measurement model are all nonlinear. But note that the multiplicative noise case is just a special case of the stochastic nonlinearities considered in [36]. Therefore, an explicit and systematic solution to this problem can be extended.

In addition, the correlation of additive noises is one of the key factors to the filtering algorithm. Disturbed by the complicated environment, the additive noises often show the characteristic of correlation in the practical application. Unluckily, the design procedures of all the above filters for multiplicative noises or packet losses are based on the assumption that there are uncorrelated additive noises in the system. In fact, this assumption does not always come into existence, and the process noise might be correlated with the measurement noise in real applications. In [38], a modified UKF for nonlinear systems with correlated additive noises is proposed. Wang et al. [39] extend the work to develop a Gaussian approximation recursive filter framework to deal with correlated noises. But model uncertainties are not considered in these works. To the best of the authors' knowledge, up to the present, based on the assumption that the process noise is correlated with the measurement noise, the nonlinear robust filtering problem with multiplicative noises and packet losses has not been reported. Therefore, in order to better reflect the actual situation and consider the complex dynamical systems, there is a strong desire to develop a robust recursive filter to handle the robust filtering problem with correlated additive noises, multiplicative noises, and packet losses.

Motivated by the above discussion, we present a robust recursive filter for a class of nonlinear systems with correlated additive noises, multiplicative noises, and packet losses. In this paper, multiplicative noises are assumed as zero mean Gaussian white noises and the packet losses are modeled as independent Bernoulli random variables. Based on the structure of the extended Kalman filter with correlated noises, the proposed filter designs an optimal upper bound of the prediction error and the filtering error covariance matrices, respectively. The main contributions of the paper are as follows. (1) In the case that the process noise is correlated with the measurement noise, a recursive filter framework is established to deal with the robust filtering problem for nonlinear systems in the presence of multiplicative noises and packet losses. (2) The addressed robust recursive filter problem is new especially when the correlated additive noises appear in the system. (3) The developed robust filter is recursive, which is suitable for online applications. The remainder of the paper is organized as follows. In Section 2, the problem is formulated. In Section 3, the robust recursive filter with correlated additive noises, multiplicative noises, and packet losses is developed. In Section 4, two simulation examples are employed, and the simulation analysis is given. In Section 5, some conclusions are drawn.

\section{Problem Formulation and Preliminaries}

Consider a general class of discrete time-varying systems with multiplicative noises, correlated additive noises, and packet losses:

$$
\begin{aligned}
& \mathbf{x}_{k+1}=f\left(\mathbf{x}_{k}\right)+\sum_{i=1}^{q} \mathbf{A}_{i k}^{s} \boldsymbol{\eta}_{i k} \mathbf{x}_{k}+\mathbf{w}_{k}, \\
& \mathbf{y}_{k}=\boldsymbol{\Sigma}_{k} h\left(\mathbf{x}_{k}\right)+\sum_{i=1}^{r} \mathbf{C}_{i k}^{s} \boldsymbol{\xi}_{i k} \mathbf{x}_{k}+\mathbf{v}_{k},
\end{aligned}
$$

where $\mathbf{x}_{k} \in \mathbf{R}^{n}$ is the state vector, $\mathbf{y}_{k} \in \mathbf{R}^{m}$ is the measurement vector, $\boldsymbol{\eta}_{i k}$ and $\boldsymbol{\xi}_{i k}$ are the uncorrelated zero mean Gaussian multiplicative noises, and $\mathbf{A}_{i k}^{s}$ and $\mathbf{C}_{i k}^{s}$ are known matrices with appropriate dimension. The diagonal matrix $\Sigma_{k}$ is denoted as $\Sigma_{k}=\operatorname{diag}\left\{\lambda_{k}^{1}, \lambda_{k}^{2}, \ldots, \lambda_{k}^{m}\right\}$, where $\lambda_{k}^{i}(i=1,2, \ldots, m)$ are independent Bernoulli random variables. It is assumed that $\lambda_{k}^{i}$ has the probability density function $p\left(\lambda_{k}^{i}\right)$ on the interval $[0,1]$ with mean $\mu_{k}^{i}$ and covariance $\left(\sigma_{k}^{i}\right)^{2}$. The process noise $\mathbf{w}_{k}$ and the measurement 
noise $\mathbf{v}_{k}$ are correlated zero mean Gaussian white noises, which satisfies

$$
\begin{gathered}
E\left(\mathbf{w}_{k}\right)=0, \quad \operatorname{cov}\left(\mathbf{w}_{k}, \mathbf{w}_{j}^{T}\right)=\mathbf{Q}_{k} \delta_{k j} \\
E\left(\mathbf{v}_{k}\right)=0, \quad \operatorname{cov}\left(\mathbf{v}_{k}, \mathbf{v}_{j}^{T}\right)=\mathbf{R}_{k} \delta_{k j} \\
\operatorname{cov}\left(\mathbf{w}_{k}, \mathbf{v}_{j}^{T}\right)=\mathbf{S}_{k} \delta_{k j} .
\end{gathered}
$$

The deterministic nonlinear functions $f\left(\mathbf{x}_{k}\right): \mathbf{R}^{n} \rightarrow \mathbf{R}^{n}$ and $h\left(\mathbf{x}_{k}\right): \mathbf{R}^{n} \rightarrow \mathbf{R}^{m}$ are known. According to the known measurement equation, we employ the assumption in [36] as the following form:

$$
\left\|h\left(\mathbf{x}_{k}\right)\right\| \leq a_{1}\left\|\mathbf{x}_{k}\right\|+a_{2}
$$

where $a_{1}$ and $a_{2}$ are the nonnegative scalars.

Because of existing correlated additive noises, for system (1)-(2), a recursive filter with correlated noises to be designed is constitutive of the following two steps including the state prediction and correction:

State prediction:

$$
\begin{gathered}
\widehat{\mathbf{x}}_{k+1 \mid k-1}=f\left(\widehat{\mathbf{x}}_{k \mid k-1}\right) \\
\widehat{\mathbf{x}}_{k+1 \mid k}=\widehat{\mathbf{x}}_{k+1 \mid k-1}+\mathbf{L}_{k}\left[\mathbf{y}_{k}-\overline{\mathbf{\Sigma}}_{k} h\left(\widehat{\mathbf{x}}_{k \mid k-1}\right)\right]
\end{gathered}
$$

State correction:

$$
\widehat{\mathbf{x}}_{k+1 \mid k+1}=\widehat{\mathbf{x}}_{k+1 \mid k}+\mathbf{K}_{k+1}\left[\mathbf{y}_{k+1}-\overline{\mathbf{\Sigma}}_{k+1} h\left(\widehat{\mathbf{x}}_{k+1 \mid k}\right)\right],
$$

where $\overline{\boldsymbol{\Sigma}}_{k}=E\left(\boldsymbol{\Sigma}_{k}\right)=\operatorname{diag}\left(\mu_{k}^{1}, \mu_{k}^{2}, \ldots, \mu_{k}^{m}\right) ; \widehat{\mathbf{x}}_{k \mid k-1}$ is the onestep state prediction at time $k-1$ with $\widehat{\mathbf{x}}_{0 \mid-1}=\widehat{\mathbf{x}}_{0 \mid 0} ; \widehat{\mathbf{x}}_{k+1 \mid k-1}$ is the two-step state prediction at time $k-1 ; \mathbf{L}_{k}$ and $\mathbf{K}_{k+1}$ are the gain parameters to be determined; $\widehat{\mathbf{x}}_{k+1 \mid k+1}$ is the state estimation at time $k+1$.

The aim of the paper is to design a recursive filter for the structures (5)-(7), which make the filtering prediction and estimation covariance have upper bounds in the presence of multiplicative noises and packet losses. Suppose that two positive definite matrices $\boldsymbol{\Xi}_{k+1 \mid k}$ and $\boldsymbol{\Xi}_{k+1 \mid k+1}$ satisfy

$$
\begin{gathered}
E\left[\left(\mathbf{x}_{k+1}-\widehat{\mathbf{x}}_{k+1 \mid k}\right)\left(\mathbf{x}_{k+1}-\widehat{\mathbf{x}}_{k+1 \mid k}\right)^{T}\right] \leq \boldsymbol{\Xi}_{k+1 \mid k}, \\
E\left[\left(\mathbf{x}_{k+1}-\widehat{\mathbf{x}}_{k+1 \mid k+1}\right)\left(\mathbf{x}_{k+1}-\widehat{\mathbf{x}}_{k+1 \mid k+1}\right)^{T}\right] \leq \boldsymbol{\Xi}_{k+1 \mid k+1} .
\end{gathered}
$$

The addressed filtering problem is that the designed filter parameters $\mathbf{L}_{k}$ and $\mathbf{K}_{k+1}$ in (5)-(7) should minimize the upper bounds $\boldsymbol{\Xi}_{k+1 \mid k}$ and $\boldsymbol{\Xi}_{k+1 \mid k+1}$.

Remark 1. In engineering applications, multiplicative noises constantly existing in the systems depend on the real state value, which results in the unknown noise variance. As discussed in [26], it should be seen as a model uncertainty. Moreover, the definition (3) shows that the process noise is correlated with the measurement noise. This requires employing a new state prediction step in (5)-(6), which is different from the state prediction of the recursive filter form in $[36,37]$. Meanwhile, the unknown prediction gain $\mathbf{L}_{k}$ does not exist in the literature $[36,37]$, which will lead to the different estimation results. Subsequently, the packet losses are described by utilizing the diagonal matrix $\Sigma_{k}$ in (2), which indicates that the different sensors have different failure rates. Since multiplicative noises, correlated additive noises, and packet losses are taken into account, the system (1)-(2) is more generalized to describe the realistic situations in engineering.

\section{Design of Robust Recursive Filter}

3.1. The Error Covariance Matrix. Denote the two-step prediction error as $\widetilde{\mathbf{x}}_{k+1 \mid k-1}=\mathbf{x}_{k+1}-\widehat{\mathbf{x}}_{k+1 \mid k-1}$ and the one-step prediction error as $\widetilde{\mathbf{x}}_{k+1 \mid k}=\mathbf{x}_{k+1}-\widehat{\mathbf{x}}_{k+1 \mid k}$. From (1), (2), (5), and (6), they can be calculated as

$$
\widetilde{\mathbf{x}}_{k+1 \mid k-1}=f\left(\mathbf{x}_{k}\right)-f\left(\widehat{\mathbf{x}}_{k \mid k-1}\right) \sum_{i=1}^{q} \mathbf{A}_{i k}^{s} \boldsymbol{\eta}_{i k} \mathbf{x}_{k}+\mathbf{w}_{k},
$$

$$
\begin{aligned}
\widetilde{\mathbf{x}}_{k+1 \mid k}= & \widetilde{\mathbf{x}}_{k+1 \mid k-1} \\
& -\mathbf{L}_{k}\left[\boldsymbol{\Sigma}_{k} h\left(\mathbf{x}_{k}\right)+\sum_{i=1}^{r} \mathbf{C}_{i k}^{s} \boldsymbol{\xi}_{i k} \mathbf{x}_{k}+\mathbf{v}_{k}-\overline{\boldsymbol{\Sigma}}_{k} h\left(\widehat{\mathbf{x}}_{k \mid k-1}\right)\right] .
\end{aligned}
$$

The nonlinear functions $f\left(\mathbf{x}_{k}\right)$ and $h\left(\mathbf{x}_{k}\right)$ can be linearized by utilizing the Taylor series expansion around $\widehat{\mathbf{x}}_{k \mid k-1}$ :

$$
\begin{aligned}
& f\left(\mathbf{x}_{k}\right)=f\left(\widehat{\mathbf{x}}_{k \mid k-1}\right)+\mathbf{A}_{k} \widetilde{\mathbf{x}}_{k \mid k-1}+o\left(\left|\widetilde{\mathbf{x}}_{k \mid k-1}\right|\right), \\
& h\left(\mathbf{x}_{k}\right)=h\left(\widehat{\mathbf{x}}_{k \mid k-1}\right)+\mathbf{C}_{k} \widetilde{\mathbf{x}}_{k \mid k-1}+\widehat{o}\left(\left|\widetilde{\mathbf{x}}_{k \mid k-1}\right|\right),
\end{aligned}
$$

where $\mathbf{A}_{k}=\partial f\left(\mathbf{x}_{k}\right) /\left.\partial \mathbf{x}_{k}\right|_{\mathbf{x}_{k}=\widehat{\mathbf{x}}_{k \mid k-1}} ; \mathbf{C}_{k}=\partial h\left(\mathbf{x}_{k}\right) /\left.\partial \mathbf{x}_{k}\right|_{\mathbf{x}_{k}=\widehat{\mathbf{x}}_{k \mid k-1}} ;$ $o\left(\left|\widetilde{\mathbf{x}}_{k \mid k-1}\right|\right)$ and $\widehat{o}\left(\left|\widetilde{\mathbf{x}}_{k \mid k-1}\right|\right)$ represent the high-order terms of the Taylor series expansion. According to the literature [26], $o\left(\left|\widetilde{\mathbf{x}}_{k \mid k-1}\right|\right)$ and $\widehat{o}\left(\left|\widetilde{\mathbf{x}}_{k \mid k-1}\right|\right)$ can be expressed as

$$
\begin{aligned}
& o\left(\left|\widetilde{\mathbf{x}}_{k \mid k-1}\right|\right)=\mathbf{B}_{k} \boldsymbol{\beta}_{k} \mathbf{E}_{k} \widetilde{\mathbf{x}}_{k \mid k-1}, \\
& \widehat{o}\left(\left|\widetilde{\mathbf{x}}_{k \mid k-1}\right|\right)=\mathbf{D}_{k} \boldsymbol{\alpha}_{k} \mathbf{E}_{k} \widetilde{\mathbf{x}}_{k \mid k-1},
\end{aligned}
$$

where $\mathbf{B}_{k} \in \mathbf{R}^{n \times n}$ and $\mathbf{D}_{k} \in \mathbf{R}^{m \times n}$ are known scaling matrices, $\mathbf{E}_{k} \in \mathbf{R}^{n \times n}$ is a known tuning matrix, and $\boldsymbol{\beta}_{k} \in \mathbf{R}^{n \times n}$ and $\boldsymbol{\alpha}_{k} \in \mathbf{R}^{n \times n}$ are unknown time-varying matrices accounting for the linearization errors of the system model that satisfies

$$
\boldsymbol{\beta}_{k} \boldsymbol{\beta}_{k}^{T} \leq \mathbf{I}, \quad \boldsymbol{\alpha}_{k} \boldsymbol{\alpha}_{k}^{T} \leq \mathbf{I}
$$

According to (9), (11), and (13), the two-step prediction error can be written as

$$
\widetilde{\mathbf{x}}_{k+1 \mid k-1}=\left(\mathbf{A}_{k}+\mathbf{B}_{k} \boldsymbol{\beta}_{k} \mathbf{E}_{k}\right) \widetilde{\mathbf{x}}_{k \mid k-1}+\sum_{i=1}^{q} \mathbf{A}_{i k}^{s} \boldsymbol{\eta}_{i k} \mathbf{x}_{k}+\mathbf{w}_{k} .
$$


Substituting (12), (13), and (15) into (9), the one-step prediction error can be determined as

$$
\begin{aligned}
\widetilde{\mathbf{x}}_{k+1 \mid k}= & \left(\mathbf{A}_{k}+\mathbf{B}_{k} \boldsymbol{\beta}_{k} \mathbf{E}_{k}\right) \widetilde{\mathbf{x}}_{k \mid k-1}+\sum_{i=1}^{q} \mathbf{A}_{i k}^{s} \boldsymbol{\eta}_{i k} \mathbf{x}_{k}+\mathbf{w}_{k} \\
& -\mathbf{L}_{k}\left[\boldsymbol{\Sigma}_{k} h\left(\mathbf{x}_{k}\right)+\sum_{i=1}^{r} \mathbf{C}_{i k}^{s} \boldsymbol{\xi}_{i k} \mathbf{x}_{k}+\mathbf{v}_{k}\right. \\
& \left.-\overline{\boldsymbol{\Sigma}}_{k}\left(h\left(\mathbf{x}_{k}\right)-\left(\mathbf{C}_{k}+\mathbf{D}_{k} \boldsymbol{\alpha}_{k} \mathbf{E}_{k}\right) \widetilde{\mathbf{x}}_{k \mid k-1}\right)\right] \\
= & {\left[\left(\mathbf{A}_{k}+\mathbf{L}_{k} \overline{\boldsymbol{\Sigma}}_{k} \mathbf{C}_{k}\right)+\left(\mathbf{B}_{k} \boldsymbol{\beta}_{k}+\mathbf{L}_{k} \overline{\boldsymbol{\Sigma}}_{k} \mathbf{D}_{k} \boldsymbol{\alpha}_{k}\right) \mathbf{E}_{k}\right] \widetilde{\mathbf{x}}_{k \mid k-1} } \\
& -\mathbf{L}_{k}\left(\boldsymbol{\Sigma}_{k}-\overline{\boldsymbol{\Sigma}}_{k}\right) h\left(\mathbf{x}_{k}\right)-\mathbf{L}_{k}\left(\sum_{i=1}^{r} \mathbf{C}_{i k}^{s} \boldsymbol{\xi}_{i k} \mathbf{x}_{k}+\mathbf{v}_{k}\right) \\
& +\sum_{i=1}^{q} \mathbf{A}_{i k}^{s} \boldsymbol{\eta}_{i k} \mathbf{x}_{k}+\mathbf{w}_{k} \\
= & {\left[\left(\mathbf{A}_{k}+\mathbf{L}_{k} \overline{\boldsymbol{\Sigma}}_{k} \mathbf{C}_{k}\right)+\mathbf{H}_{k} \mathbf{F}_{k} \mathbf{J}_{k} \mathbf{E}_{k}\right] \widetilde{\mathbf{x}}_{k \mid k-1} } \\
& -\mathbf{L}_{k}\left(\boldsymbol{\Sigma}_{k}-\overline{\boldsymbol{\Sigma}}_{k}\right) h\left(\mathbf{x}_{k}\right)-\mathbf{L}_{k}\left(\sum_{i=1}^{r} \mathbf{C}_{i k}^{s} \boldsymbol{\xi}_{i k} \mathbf{x}_{k}+\mathbf{v}_{k}\right) \\
& +\sum_{i=1}^{q} \mathbf{A}_{i k}^{s} \boldsymbol{\eta}_{i k} \mathbf{x}_{k}+\mathbf{w}_{k},
\end{aligned}
$$

where it is assumed that $\mathbf{H}_{k}=\left[\begin{array}{ll}\mathbf{B}_{k} & \mathbf{L}_{k} \overline{\boldsymbol{\Sigma}}_{k} \mathbf{D}_{k}\end{array}\right], \mathbf{F}_{k}=\left[\begin{array}{cc}\boldsymbol{\beta}_{k} & \mathbf{0}_{n \times n} \\ \mathbf{0}_{n \times n} & \boldsymbol{\alpha}_{k}\end{array}\right]$, and $\mathbf{J}_{k}=\left[\begin{array}{c}\mathbf{I}_{\times \times n} \\ \mathbf{I}_{n \times n}\end{array}\right]$ and from (14) we have $\mathbf{F}_{k} \mathbf{F}_{k}^{T} \leq \mathbf{I}$.

as

The one-step prediction error covariance can be obtained

$$
\begin{aligned}
\mathbf{P}_{k+1 \mid k}= & E\left(\widetilde{\mathbf{x}}_{k+1 \mid k} \widetilde{\mathbf{x}}_{k+1 \mid k}^{T}\right) \\
= & {\left[\left(\mathbf{A}_{k}+\mathbf{L}_{k} \overline{\mathbf{\Sigma}}_{k} \mathbf{C}_{k}\right)+\mathbf{H}_{k} \mathbf{F}_{k} \mathbf{J}_{k} \mathbf{E}_{k}\right] \mathbf{P}_{k \mid k-1} } \\
& \times\left[\left(\mathbf{A}_{k}+\mathbf{L}_{k} \overline{\mathbf{s}}_{k} \mathbf{C}_{k}\right)+\mathbf{H}_{k} \mathbf{F}_{k} \mathbf{J}_{k} \mathbf{E}_{k}\right]^{T}-\mathbf{S}_{k} \mathbf{L}_{k}^{T}-\mathbf{L}_{k} \mathbf{S}_{k}^{T} \\
& +\mathbf{L}_{k}\left[\breve{\mathbf{\Sigma}}_{k} \circ E\left(h\left(\mathbf{x}_{k}\right) h^{T}\left(\mathbf{x}_{k}\right)\right)\right. \\
& \left.\quad+\sum_{i=1}^{r} \mathbf{C}_{i k}^{s} E\left(\mathbf{x}_{k} \mathbf{x}_{k}^{T}\right)\left(\mathbf{C}_{i k}^{s}\right)^{T}+\mathbf{R}_{k}\right] \mathbf{L}_{k}^{T} \\
& +\sum_{i=1}^{q} \mathbf{A}_{i k}^{s} E\left(\mathbf{x}_{k} \mathbf{x}_{k}^{T}\right)\left(\mathbf{A}_{i k}^{s}\right)^{T}+\mathbf{Q}_{k},
\end{aligned}
$$

where $\breve{\boldsymbol{\Sigma}}_{k}=\operatorname{diag}\left\{\left(\sigma_{k}^{1}\right)^{2},\left(\sigma_{k}^{2}\right)^{2}, \ldots,\left(\sigma_{k}^{m}\right)^{2}\right\}$.

Denote the estimation error as

$$
\widetilde{\mathbf{x}}_{k+1 \mid k+1}=\mathbf{x}_{k+1}-\widehat{\mathbf{x}}_{k+1 \mid k+1} .
$$

From (1), (2), and (7), it can be rewritten as

$$
\begin{aligned}
& \widetilde{\mathbf{x}}_{k+1 \mid k+1} \\
& =\widetilde{\mathbf{x}}_{k+1 \mid k}-\mathbf{K}_{k+1}\left[\mathbf{y}_{k+1}-\overline{\mathbf{\Sigma}}_{k+1} h\left(\widehat{\mathbf{x}}_{k+1 \mid k}\right)\right] \\
& =\widetilde{\mathbf{x}}_{k+1 \mid k} \\
& \quad-\mathbf{K}_{k+1}\left[\boldsymbol{\Sigma}_{k+1} h\left(\mathbf{x}_{k+1}\right)+\sum_{i=1}^{r} \mathbf{C}_{i k+1}^{s} \boldsymbol{\xi}_{i k+1} \mathbf{x}_{k+1}+\mathbf{v}_{k+1}\right. \\
& \left.\quad-\overline{\boldsymbol{\Sigma}}_{k+1} h\left(\widehat{\mathbf{x}}_{k+1 \mid k}\right)\right] \\
& =\left(\mathbf{I}-\mathbf{K}_{k+1} \overline{\boldsymbol{\Sigma}}_{k+1} \overline{\mathbf{C}}_{k+1}-\mathbf{K}_{k+1} \overline{\boldsymbol{\Sigma}}_{k+1} \overline{\mathbf{D}}_{k+1} \overline{\boldsymbol{\alpha}}_{k+1} \overline{\mathbf{E}}_{k+1}\right) \widetilde{\mathbf{x}}_{k+1 \mid k} \\
& \quad-\mathbf{K}_{k+1}\left(\sum_{i=1}^{r} \mathbf{C}_{i k+1}^{s} \boldsymbol{\xi}_{i k+1} \mathbf{x}_{k+1}+\mathbf{v}_{k+1}\right) \\
& \quad-\mathbf{K}_{k+1}\left(\boldsymbol{\Sigma}_{k+1}-\overline{\boldsymbol{\Sigma}}_{k+1}\right) h\left(\mathbf{x}_{k+1}\right),
\end{aligned}
$$

where $\overline{\mathbf{C}}_{k+1}=\partial h\left(\mathbf{x}_{k}\right) /\left.\partial \mathbf{x}_{k}\right|_{\mathbf{x}_{k}=\widehat{\mathbf{x}}_{k+1 k}} ; \overline{\mathbf{D}}_{k+1}$ is a known problemdependent scaling matrix; $\overline{\mathbf{E}}_{k+1}$ is a known tuning matrix; $\overline{\boldsymbol{\alpha}}_{k+1}$ is an unknown time-varying matrix that satisfies

$$
\overline{\boldsymbol{\alpha}}_{k+1} \overline{\boldsymbol{\alpha}}_{k+1}^{T} \leq \mathbf{I} .
$$

Subsequently, in the light of (19), the filtering error covariance can be expressed as

$$
\begin{aligned}
& \mathbf{P}_{k+1 \mid k+1} \\
& =E\left(\widetilde{\mathbf{x}}_{k+1 \mid k+1} \widetilde{\mathbf{x}}_{k+1 \mid k+1}^{T}\right) \\
& =\left(\mathbf{I}-\mathbf{K}_{k+1} \overline{\boldsymbol{\Sigma}}_{k+1} \overline{\mathbf{C}}_{k+1}-\mathbf{K}_{k+1} \overline{\boldsymbol{\Sigma}}_{k+1} \overline{\mathbf{D}}_{k+1} \overline{\boldsymbol{\alpha}}_{k+1} \overline{\mathbf{E}}_{k+1}\right) \\
& \quad \times \mathbf{P}_{k+1 \mid k}\left(\mathbf{I}-\mathbf{K}_{k+1} \overline{\boldsymbol{\Sigma}}_{k+1} \overline{\mathbf{C}}_{k+1}-\mathbf{K}_{k+1} \overline{\boldsymbol{\Sigma}}_{k+1} \overline{\mathbf{D}}_{k+1} \overline{\boldsymbol{\alpha}}_{k+1} \overline{\mathbf{E}}_{k+1}\right)^{T} \\
& \quad+\mathbf{K}_{k+1}\left[\breve{\boldsymbol{\Sigma}}_{k+1} \circ E\left(h\left(\mathbf{x}_{k+1}\right) h^{T}\left(\mathbf{x}_{k+1}\right)\right)\right. \\
& \quad+\sum_{i=1}^{r} \mathbf{C}_{i k+1}^{s} E\left(\mathbf{x}_{k+1} \mathbf{x}_{k+1}^{T}\right)\left(\mathbf{C}_{i k+1}^{s}\right)^{T} \\
& \left.\quad+\mathbf{R}_{k+1}\right] \mathbf{K}_{k+1}^{T} .
\end{aligned}
$$

Remark 2. Since there are the high-order errors, the matrices $\boldsymbol{\beta}_{k}, \boldsymbol{\alpha}_{k}$, and $\overline{\boldsymbol{\alpha}}_{k+1}$ are unknown, which makes the fact that the prediction covariance $\mathbf{P}_{k+1 \mid k}$ and the filtering error covariance $\mathbf{P}_{k+1 \mid k+1}$ from (17) and (21) cannot be computed directly. In order to complete the design of the filter, an effective way is to calculate the upper bounds for the $\mathbf{P}_{k+1 \mid k}$ and $\mathbf{P}_{k+1 \mid k+1}$ and then design the prediction gain $\mathbf{L}_{k}$ and the filtering gain $\mathbf{K}_{k+1}$ to minimize the upper bounds. Due to the influence correlated noises and unknown prediction gain $\mathbf{L}_{k}$, there is a striking contrast between the prediction covariance $\mathbf{P}_{k+1 \mid k}$ in this paper and the counterpart in the literature $[36,37]$. 
3.2. The Robust Recursive Filter Design. To develop the robust recursive filter, the following lemmas are given.

Lemma 3 (see $[40]$ ). Let $\mathbf{A}=\left[a_{i j}\right]_{n \times n}$ be a real matrix and let $\mathbf{B}=\operatorname{diag}\left(b_{1}, b_{2}, \ldots, b_{n}\right)$ be a diagonal random matrix. Then

$$
E\left\{\mathbf{B A B} \mathbf{B}^{T}\right\}=\left[\begin{array}{cccc}
E\left\{b_{1}^{2}\right\} & E\left\{b_{1} b_{2}\right\} & \cdots & E\left\{b_{1} b_{n}\right\} \\
E\left\{b_{2} b_{1}\right\} & E\left\{b_{2}^{2}\right\} & \cdots & E\left\{b_{2} b_{n}\right\} \\
\vdots & \vdots & \ddots & \vdots \\
E\left\{b_{n} b_{1}\right\} & E\left\{b_{n} b_{2}\right\} & \cdots & E\left\{b_{n}^{2}\right\}
\end{array}\right] \circ \mathbf{A}
$$

where $\circ$ is the Hadamard product.

Lemma 4 (see [41]). Given matrices $\mathbf{A}, \mathbf{H}, \mathbf{E}$, and $\mathbf{F}$ with compatible dimensions such that $\mathbf{F F}^{T} \leq \mathbf{I}$, let $\mathbf{X}$ be a symmetric positive definite matrix and let $\gamma$ be an arbitrary positive constant such that

$$
\gamma^{-1} \mathbf{I}-\mathbf{E X E}^{T}>0
$$

Then the following matrix inequality holds:

$$
\begin{gathered}
(\mathbf{A}+\mathbf{H F E}) \mathbf{X}(\mathbf{A}+\mathbf{H F E})^{T} \leq \mathbf{A}\left(\mathbf{X}^{-1}-\gamma \mathbf{E}^{T} \mathbf{E}\right)^{-1} \mathbf{A}^{T} \\
+\gamma^{-1} \mathbf{H} \mathbf{H}^{T} .
\end{gathered}
$$

Lemma 5 (see [42]). For $0 \leq k \leq n$, suppose that $\mathbf{X}=\mathbf{X}^{T}>0$, $\mathbf{e}_{k}(\mathbf{X})=\mathbf{e}_{k}^{T}(\mathbf{X}) \in \mathbf{R}^{n \times n}$, and $\mathbf{g}_{k}(\mathbf{X})=\mathbf{g}_{k}^{T}(\mathbf{X}) \in \mathbf{R}^{n \times n}$. If there exists $\mathbf{Y} \geq \mathbf{X}$ such that

$$
\begin{aligned}
& \mathbf{e}_{k}(\mathbf{Y}) \geq \mathbf{e}_{k}(\mathbf{X}), \\
& \mathbf{g}_{k}(\mathbf{Y})>\mathbf{e}_{k}(\mathbf{Y}),
\end{aligned}
$$

then the solutions $\mathbf{M}_{k}$ and $\mathbf{N}_{k}$ to the following difference equations,

$$
\begin{gathered}
\mathbf{M}_{k+1}=\mathbf{e}_{k}\left(\mathbf{M}_{k}\right), \quad \mathbf{N}_{k+1}=\mathbf{g}_{k}\left(\mathbf{N}_{k}\right), \\
\mathbf{M}_{0}=\mathbf{N}_{0}>0
\end{gathered}
$$

satisfy $\mathbf{M}_{k} \leq \mathbf{N}_{k}$.

According to these lemmas, the following theorem is given to obtain the main results of the robust recursive filter.

Theorem 6. Consider the covariance matrices of the one-step prediction errorand the filtering error in (17) and (21). Assume that the conditions shown in (14) and (20) come into existence.
Let $\lambda_{1}, \lambda_{2}, \varepsilon_{1}$, and $\varepsilon_{2}$ be positive scalars. If the following two discrete-time Riccati difference equations,

$$
\begin{aligned}
& \boldsymbol{\Xi}_{k+1 \mid k} \\
& =\left(\mathbf{A}_{k}+\mathbf{L}_{k} \overline{\boldsymbol{\Sigma}}_{k} \mathbf{C}_{k}\right)\left(\boldsymbol{\Xi}_{k \mid k-1}^{-1}-2 \lambda_{1} \mathbf{E}_{k}^{T} \mathbf{E}_{k}\right)^{-1}\left(\mathbf{A}_{k}+\mathbf{L}_{k} \overline{\boldsymbol{\Sigma}}_{k} \mathbf{C}_{k}\right)^{T} \\
& +\lambda_{1}^{-1}\left(\mathbf{B}_{k} \mathbf{B}_{k}^{T}+\mathbf{L}_{k} \overline{\boldsymbol{\Sigma}}_{k} \mathbf{D}_{k} \mathbf{D}_{k}^{T} \overline{\boldsymbol{\Sigma}}_{k}^{T} \mathbf{L}_{k}^{T}\right)+\mathbf{Q}_{k}-\mathbf{S}_{k} \mathbf{L}_{k}^{T}-\mathbf{L}_{k} \mathbf{S}_{k}^{T} \\
& +\mathbf{L}_{k}\left\{\breve{\boldsymbol{\Sigma}}_{k} \circ\left[2\left(a_{1}^{2} \operatorname{tr}\left(\boldsymbol{\Omega}_{k \mid k-1}\right)+a_{2}^{2}\right)\right] \mathbf{I}\right. \\
& \left.+\sum_{i=1}^{r} \mathbf{C}_{i k}^{s} \boldsymbol{\Omega}_{k \mid k-1}\left(\mathbf{C}_{i k}^{s}\right)^{T}+\mathbf{R}_{k}\right\} \mathbf{L}_{k}^{T}+\sum_{i=1}^{q} \mathbf{A}_{i k}^{s} \boldsymbol{\Omega}_{k \mid k-1}\left(\mathbf{A}_{i k}^{s}\right)^{T}, \\
& \Xi_{k+1 \mid k+1} \\
& =\left(\mathbf{I}-\mathbf{K}_{k+1} \overline{\mathbf{\Sigma}}_{k+1} \overline{\mathbf{C}}_{k+1}\right)\left(\boldsymbol{\Xi}_{k+1 \mid k}^{-1}-\lambda_{2} \overline{\mathbf{E}}_{k+1}^{T} \overline{\mathbf{E}}_{k+1}\right)^{-1} \\
& \times\left(\mathbf{I}-\mathbf{K}_{k+1} \bar{\Sigma}_{k+1} \overline{\mathbf{C}}_{k+1}\right)^{T}+\lambda_{2}^{-1} \mathbf{K}_{k+1} \overline{\boldsymbol{\Sigma}}_{k+1} \overline{\mathbf{D}}_{k+1} \overline{\mathbf{D}}_{k+1}^{T} \overline{\mathbf{\Sigma}}_{k+1}^{T} \mathbf{K}_{k+1}^{T} \\
& +\mathbf{K}_{k+1}\left\{\breve{\mathbf{\Sigma}}_{k+1} \circ\left[2\left(a_{1}^{2} \operatorname{tr}\left(\Delta_{k+1 \mid k}\right)+a_{2}^{2}\right)\right] \mathbf{I}\right. \\
& \left.+\sum_{i=1}^{r} \mathbf{C}_{i k+1}^{s} \Delta_{k+1 \mid k}\left(\mathbf{C}_{i k+1}^{s}\right)^{T}+\mathbf{R}_{k+1}\right\} \mathbf{K}_{k+1}^{T}
\end{aligned}
$$

with initial covariance $\Xi_{0 \mid-1}=\Xi_{0 \mid 0}>0$ have positive definite solution, such that for $0 \leq k \leq N$

$$
\begin{gathered}
\lambda_{1}^{-1} \mathbf{I}-\mathbf{J}_{k} \mathbf{E}_{k} \boldsymbol{\Xi}_{k \mid k-1}\left(\mathbf{J}_{k} \mathbf{E}_{k}\right)^{T}>0, \\
\lambda_{2}^{-1} \mathbf{I}-\overline{\mathbf{E}}_{k+1} \boldsymbol{\Xi}_{k+1 \mid k} \overline{\mathbf{E}}_{k+1}^{T}>0,
\end{gathered}
$$

where

$$
\begin{aligned}
& \boldsymbol{\Omega}_{k \mid k-1}=\left(1+\varepsilon_{1}\right) \boldsymbol{\Xi}_{k \mid k-1}+\left(1+\varepsilon_{1}^{-1}\right) \widehat{\mathbf{x}}_{k \mid k-1} \widehat{\mathbf{x}}_{k \mid k-1}^{T}, \\
& \boldsymbol{\Delta}_{k+1 \mid k}=\left(1+\varepsilon_{2}\right) \boldsymbol{\Xi}_{k+1 \mid k}+\left(1+\varepsilon_{2}^{-1}\right) \widehat{\mathbf{x}}_{k+1 \mid k} \widehat{\mathbf{x}}_{k+1 \mid k}^{T},
\end{aligned}
$$

then the prediction gain $\mathbf{L}_{k}$ and the filtering gain $\mathbf{K}_{k+1}$ are given by

$$
\begin{aligned}
\mathbf{L}_{k}= & {\left[\mathbf{S}_{k}-\mathbf{A}_{k}\left(\boldsymbol{\Xi}_{k \mid k-1}^{-1}-2 \lambda_{1} \mathbf{E}_{k}^{T} \mathbf{E}_{k}\right)^{-1} \mathbf{C}_{k}^{T} \overline{\boldsymbol{\Sigma}}_{k}\right] } \\
\times & \left\{\overline{\boldsymbol{\Sigma}}_{k} \mathbf{C}_{k}\left(\boldsymbol{\Xi}_{k \mid k-1}^{-1}-2 \lambda_{1} \mathbf{E}_{k}^{T} \mathbf{E}_{k}\right)^{-1} \mathbf{C}_{k}^{T} \overline{\boldsymbol{\Sigma}}_{k}\right. \\
& +\lambda_{1}^{-1} \overline{\boldsymbol{\Sigma}}_{k} \mathbf{D}_{k} \mathbf{D}_{k}^{T} \overline{\boldsymbol{\Sigma}}_{k}^{T}
\end{aligned}
$$




$$
\begin{aligned}
& +\breve{\boldsymbol{\Sigma}}_{k} \circ\left[2\left(a_{1}^{2} \operatorname{tr}\left(\boldsymbol{\Omega}_{k \mid k-1}\right)+a_{2}^{2}\right)\right] \mathbf{I} \\
& \left.+\sum_{i=1}^{r} \mathbf{C}_{i k}^{s} \boldsymbol{\Omega}_{k \mid k-1}\left(\mathbf{C}_{i k}^{s}\right)^{T}+\mathbf{R}_{k}\right\}^{-1}, \\
& \mathbf{K}_{k+1}=\left(\boldsymbol{\Xi}_{k+1 \mid k}^{-1}-\lambda_{2} \overline{\mathbf{E}}_{k+1}^{T} \overline{\mathbf{E}}_{k+1}\right)^{-1} \overline{\mathbf{C}}_{k+1}^{T} \overline{\boldsymbol{\Sigma}}_{k+1} \\
& \times\left\{\overline{\boldsymbol{\Sigma}}_{k+1}^{-} \overline{\mathbf{C}}_{k+1}\left(\boldsymbol{\Xi}_{k+1 \mid k}^{-1}-\lambda_{2} \overline{\mathbf{E}}_{k+1}^{T} \overline{\mathbf{E}}_{k+1}\right)^{-1} \overline{\mathbf{C}}_{k+1}^{T} \overline{\boldsymbol{\Sigma}}_{k+1}\right. \\
& +\lambda_{2}^{-1} \overline{\boldsymbol{\Sigma}}_{k+1} \overline{\mathbf{D}}_{k+1} \overline{\mathbf{D}}_{k+1}^{T} \overline{\boldsymbol{\Sigma}}_{k+1}^{T}+\sum_{i=1}^{r} \mathbf{C}_{i k+1}^{s} \Delta_{k+1 \mid k}\left(\mathbf{C}_{i k+1}^{s}\right)^{T} \\
& \left.+\breve{\boldsymbol{\Sigma}}_{k+1} \circ\left[2\left(a_{1}^{2} \operatorname{tr}\left(\boldsymbol{\Delta}_{k+1 \mid k}\right)+a_{2}^{2}\right)\right] \mathbf{I}+\mathbf{R}_{k+1}\right\}^{-1}
\end{aligned}
$$

and the matrices $\boldsymbol{\Xi}_{k+1 \mid k}$ and $\boldsymbol{\Xi}_{k+1 \mid k+1}$ are upper bounds for $\mathbf{P}_{k+1 \mid k}$ and $\mathbf{P}_{k+1 \mid k+1}$; that is,

$$
\mathbf{P}_{k+1 \mid k} \leq \boldsymbol{\Xi}_{k+1 \mid k}, \quad \mathbf{P}_{k+1 \mid k+1} \leq \boldsymbol{\Xi}_{k+1 \mid k+1} .
$$

Moreover, the prediction gain $\mathbf{L}_{k}$ given by (31) minimizes the upper bound $\boldsymbol{\Xi}_{k+1 \mid k}$ and the filtering gain $\mathbf{K}_{k+1}$ given by (32) minimizes the upper bound $\boldsymbol{\Xi}_{k+1 \mid k+1}$.

Proof. According to (17) and (21), the prediction covariance $\mathbf{P}_{k+1 \mid k}$ and the filtering error covariance $\mathbf{P}_{k+1 \mid k+1}$ can be expressed as the functions of $\mathbf{P}_{k \mid k-1}$ and $\mathbf{P}_{k+1 \mid k}$ :

$$
\begin{aligned}
\mathbf{P}_{k+1 \mid k} & =\mathbf{P}_{k+1 \mid k}\left(\mathbf{P}_{k \mid k-1}\right), \\
\mathbf{P}_{k+1 \mid k+1} & =\mathbf{P}_{k+1 \mid k+1}\left(\mathbf{P}_{k+1 \mid k}\right) .
\end{aligned}
$$

Assume that $\varepsilon_{1}$ is a positive scalar. The matrix inequality can be obtained as

$$
\widetilde{\mathbf{x}}_{k \mid k-1} \widehat{\mathbf{x}}_{k \mid k-1}^{T}+\widehat{\mathbf{x}}_{k \mid k-1} \widetilde{\mathbf{x}}_{k \mid k-1}^{T} \leq \varepsilon_{1} \widetilde{\mathbf{x}}_{k \mid k-1} \widetilde{\mathbf{x}}_{k \mid k-1}^{T}+\varepsilon_{1}^{-1} \widehat{\mathbf{x}}_{k \mid k-1} \widehat{\mathbf{x}}_{k \mid k-1}^{T} .
$$

From (35), we have

$$
\begin{aligned}
E\left(\mathbf{x}_{k} \mathbf{x}_{k}^{T}\right) & \leq E\left[\left(\widetilde{\mathbf{x}}_{k \mid k-1}+\widehat{\mathbf{x}}_{k \mid k-1}\right)\left(\widetilde{\mathbf{x}}_{k \mid k-1}+\widehat{\mathbf{x}}_{k \mid k-1}\right)^{T}\right] \\
& \leq\left(1+\varepsilon_{1}\right) \mathbf{P}_{k \mid k-1}+\left(1+\varepsilon_{1}^{-1}\right) \widehat{\mathbf{x}}_{k \mid k-1} \widehat{\mathbf{x}}_{k \mid k-1}^{T} \\
& =\boldsymbol{\Psi}_{k \mid k-1} .
\end{aligned}
$$

According to the literature [36], based on (4), we obtain

$$
\begin{aligned}
E\left\{h\left(\mathbf{x}_{k}\right) h^{T}\left(\mathbf{x}_{k}\right)\right\} & \leq E\left\{\left\|h\left(\mathbf{x}_{k}\right)\right\|^{2}\right\} \mathbf{I} \leq E\left\{\left(a_{1}\left\|\mathbf{x}_{k}\right\|+a_{2}\right)^{2}\right\} \mathbf{I} \\
& \leq\left(2 a_{1}^{2} E\left\{\left\|\mathbf{x}_{k}\right\|^{2}\right\}+2 a_{2}^{2}\right) \mathbf{I} \\
& =2\left[a_{1}^{2} \operatorname{tr}\left(E\left(\mathbf{x}_{k} \mathbf{x}_{k}^{T}\right)\right)+a_{2}^{2}\right] \mathbf{I} .
\end{aligned}
$$

Substituting (36) into (37), it can be rewritten as

$$
E\left\{h\left(\mathbf{x}_{k}\right) h^{T}\left(\mathbf{x}_{k}\right)\right\} \leq 2\left[a_{1}^{2} \operatorname{tr}\left(\Psi_{k \mid k-1}\right)+a_{2}^{2}\right] \mathbf{I} .
$$

Furthermore, inserting (36) and (38) into (17), the onestep prediction error covariance can be rearranged as

$$
\begin{aligned}
\mathbf{P}_{k+1 \mid k} \leq & {\left[\left(\mathbf{A}_{k}+\mathbf{L}_{k} \overline{\boldsymbol{\Sigma}}_{k} \mathbf{C}_{k}\right)+\mathbf{H}_{k} \mathbf{F}_{k} \mathbf{J}_{k} \mathbf{E}_{k}\right] \mathbf{P}_{k \mid k-1} } \\
& \times\left[\left(\mathbf{A}_{k}+\mathbf{L}_{k} \overline{\boldsymbol{\Sigma}}_{k} \mathbf{C}_{k}\right)+\mathbf{H}_{k} \mathbf{F}_{k} \mathbf{J}_{k} \mathbf{E}_{k}\right]^{T}-\mathbf{S}_{k} \mathbf{L}_{k}^{T}-\mathbf{L}_{k} \mathbf{S}_{k}^{T} \\
+ & \mathbf{L}_{k}\left[\breve{\boldsymbol{\Sigma}}_{k} \circ 2\left[a_{1}^{2} \operatorname{tr}\left(\boldsymbol{\Psi}_{k \mid k-1}\right)+a_{2}^{2}\right] \mathbf{I}\right. \\
& \left.+\sum_{i=1}^{r} \mathbf{C}_{i k}^{s} \boldsymbol{\Psi}_{k \mid k-1}\left(\mathbf{C}_{i k}^{s}\right)^{T}+\mathbf{R}_{k}\right\} \mathbf{L}_{k}^{T} \\
& +\sum_{i=1}^{q} \mathbf{A}_{i k}^{s} \boldsymbol{\Psi}_{k \mid k-1}\left(\mathbf{A}_{i k}^{s}\right)^{T}+\mathbf{Q}_{k},
\end{aligned}
$$

Similar to (36) and (38), let $\varepsilon_{2}$ be a positive scalar; we have

$$
\begin{aligned}
E\left(\mathbf{x}_{k+1} \mathbf{x}_{k+1}^{T}\right) & \leq\left(1+\varepsilon_{2}\right) \mathbf{P}_{k+1 \mid k}+\left(1+\varepsilon_{2}^{-1}\right) \widehat{\mathbf{x}}_{k+1 \mid k} \widehat{\mathbf{x}}_{k+1 \mid k}^{T} \\
& =\boldsymbol{\Lambda}_{k+1 \mid k}, \\
E\left\{h\left(\mathbf{x}_{k+1}\right)\right. & \left.h^{T}\left(\mathbf{x}_{k+1}\right)\right\} \leq 2\left[a_{1}^{2} \operatorname{tr}\left(\boldsymbol{\Lambda}_{k+1 \mid k}\right)+a_{2}^{2}\right] \mathbf{I} .
\end{aligned}
$$

Substituting (40) into (21), we have

$$
\begin{gathered}
\mathbf{P}_{k+1 \mid k+1} \\
=\left(\mathbf{I}-\mathbf{K}_{k+1} \overline{\boldsymbol{\Sigma}}_{k+1} \overline{\mathbf{C}}_{k+1}-\mathbf{K}_{k+1} \overline{\boldsymbol{\Sigma}}_{k+1} \overline{\mathbf{D}}_{k+1} \overline{\boldsymbol{\alpha}}_{k+1} \overline{\mathbf{E}}_{k+1}\right) \mathbf{P}_{k+1 \mid k} \\
\times\left(\mathbf{I}-\mathbf{K}_{k+1} \overline{\boldsymbol{\Sigma}}_{k+1} \overline{\mathbf{C}}_{k+1}-\mathbf{K}_{k+1} \overline{\boldsymbol{\Sigma}}_{k+1} \overline{\mathbf{D}}_{k+1} \overline{\boldsymbol{\alpha}}_{k+1} \overline{\mathbf{E}}_{k+1}\right)^{T} \\
+\mathbf{K}_{k+1}\left\{\breve{\boldsymbol{\Sigma}}_{k+1} \circ\left[2\left(a_{1}^{2} \operatorname{tr}\left(\boldsymbol{\Lambda}_{k+1 \mid k}\right)+a_{2}^{2}\right)\right] \mathbf{I}\right. \\
\left.+\sum_{i=1}^{r} \mathbf{C}_{i k+1}^{s} \boldsymbol{\Lambda}_{k+1 \mid k}\left(\mathbf{C}_{i k+1}^{s}\right)^{T}+\mathbf{R}_{k+1}\right\} \mathbf{K}_{k+1}^{T} .
\end{gathered}
$$

From (27) and (28), $\boldsymbol{\Xi}_{k+1 \mid k}$ and $\boldsymbol{\Xi}_{k+1 \mid k+1}$ can be rewritten as the functions of $\boldsymbol{\Xi}_{k \mid k-1}$ and $\boldsymbol{\Xi}_{k+1 \mid k}$ :

$$
\begin{aligned}
\boldsymbol{\Xi}_{k+1 \mid k} & =\boldsymbol{\Xi}_{k+1 \mid k}\left(\boldsymbol{\Xi}_{k \mid k-1}\right), \\
\boldsymbol{\Xi}_{k+1 \mid k+1} & =\boldsymbol{\Xi}_{k+1 \mid k+1}\left(\boldsymbol{\Xi}_{k+1 \mid k}\right) .
\end{aligned}
$$

Assume that there exist $\lambda_{1}>0$ and $\lambda_{2}>0$. Let the matrices $\mathbf{E}_{k}$ and $\overline{\mathbf{E}}_{k+1}$ satisfy the inequalities

$$
\begin{gathered}
\lambda_{1}^{-1} \mathbf{I}-\mathbf{J}_{k} \mathbf{E}_{k} \boldsymbol{\Xi}_{k \mid k-1}\left(\mathbf{J}_{k} \mathbf{E}_{k}\right)^{T}>0, \\
\lambda_{2}^{-1} \mathbf{I}-\overline{\mathbf{E}}_{k+1} \boldsymbol{\Xi}_{k+1 \mid k} \overline{\mathbf{E}}_{k+1}^{T}>0 .
\end{gathered}
$$


According to Lemma 4, we have

$$
\begin{aligned}
& {\left[\left(\mathbf{A}_{k}+\mathbf{L}_{k} \overline{\boldsymbol{\Sigma}}_{k} \mathbf{C}_{k}\right)+\mathbf{H}_{k} \mathbf{F}_{k} \mathbf{J}_{k} \mathbf{E}_{k}\right] \mathbf{\Xi}_{k \mid k-1}} \\
& \times\left[\left(\mathbf{A}_{k}+\mathbf{L}_{k} \overline{\boldsymbol{\Sigma}}_{k} \mathbf{C}_{k}\right)+\mathbf{H}_{k} \mathbf{F}_{k} \mathbf{J}_{k} \mathbf{E}_{k}\right]^{T} \\
& \leq\left(\mathbf{A}_{k}+\mathbf{L}_{k} \overline{\boldsymbol{\Sigma}}_{k} \mathbf{C}_{k}\right)\left(\mathbf{\Xi}_{k \mid k-1}^{-1}-2 \lambda_{1} \mathbf{E}_{k}^{T} \mathbf{E}_{k}\right)^{-1} \\
& \quad \times\left(\mathbf{A}_{k}+\mathbf{L}_{k} \overline{\boldsymbol{\Sigma}}_{k} \mathbf{C}_{k}\right)^{T}+\lambda_{1}^{-1}\left(\mathbf{B}_{k} \mathbf{B}_{k}^{T}+\mathbf{L}_{k} \overline{\boldsymbol{\Sigma}}_{k} \mathbf{D}_{k} \mathbf{D}_{k}^{T} \overline{\boldsymbol{\Sigma}}_{k}^{T} \mathbf{L}_{k}^{T}\right), \\
& \left(\mathbf{I}-\mathbf{K}_{k+1} \overline{\boldsymbol{\Sigma}}_{k+1} \overline{\mathbf{C}}_{k+1}-\mathbf{K}_{k+1} \overline{\boldsymbol{\Sigma}}_{k+1} \overline{\mathbf{D}}_{k+1} \overline{\boldsymbol{\alpha}}_{k+1} \overline{\mathbf{E}}_{k+1}\right) \mathbf{\Xi}_{k+1 \mid k} \\
& \times\left(\mathbf{I}-\mathbf{K}_{k+1} \overline{\boldsymbol{\Sigma}}_{k+1} \overline{\mathbf{C}}_{k+1}-\mathbf{K}_{k+1} \overline{\boldsymbol{\Sigma}}_{k+1} \overline{\mathbf{D}}_{k+1} \overline{\boldsymbol{\alpha}}_{k+1} \overline{\mathbf{E}}_{k+1}\right)^{T} \\
& \leq\left(\mathbf{I}-\mathbf{K}_{k+1} \overline{\boldsymbol{\Sigma}}_{k+1} \overline{\mathbf{C}}_{k+1}\right)\left(\mathbf{\Xi}_{k+1 \mid k}^{-1}-\lambda_{2} \overline{\mathbf{E}}_{k+1}^{T} \overline{\mathbf{E}}_{k+1}\right)^{-1} \\
& \quad \times\left(\mathbf{I}-\mathbf{K}_{k+1} \overline{\boldsymbol{\Sigma}}_{k+1} \overline{\mathbf{C}}_{k+1}\right)^{T} \\
& \quad+\lambda_{2}^{-1} \mathbf{K}_{k+1} \overline{\boldsymbol{\Sigma}}_{k+1} \overline{\mathbf{D}}_{k+1} \overline{\mathbf{D}}_{k+1}^{T} \overline{\boldsymbol{\Sigma}}_{k+1}^{T} \mathbf{K}_{k+1}^{T} .
\end{aligned}
$$

Combining (39) and (41)-(44), the condition (25) can be satisfied in Lemma 5. Thus, according to Lemmas 4 and 5, we have

$$
\mathbf{P}_{k+1 \mid k} \leq \boldsymbol{\Xi}_{k+1 \mid k}, \quad \mathbf{P}_{k+1 \mid k+1} \leq \boldsymbol{\Xi}_{k+1 \mid k+1} .
$$

To minimize the upper bounds, constructingthe optimized prediction gain $\mathbf{L}_{k}$ and the filtering gain $\mathbf{K}_{k+1}$ is to minimize the upper bounds $\boldsymbol{\Xi}_{k+1 \mid k}$ and $\boldsymbol{\Xi}_{k+1 \mid k+1}$; according to (27) and (28), we have

$$
\begin{aligned}
& \frac{\partial \operatorname{tr}\left(\boldsymbol{\Xi}_{k+1 \mid k}\right)}{\partial \mathbf{L}_{k}}=2\left(\mathbf{A}_{k}+\mathbf{L}_{k} \overline{\boldsymbol{\Sigma}}_{k} \mathbf{C}_{k}\right)\left(\boldsymbol{\Xi}_{k \mid k-1}^{-1}-2 \lambda_{1} \mathbf{E}_{k}^{T} \mathbf{E}_{k}\right)^{-1} \\
& \times \mathbf{C}_{k}^{T} \overline{\boldsymbol{\Sigma}}_{k}-2 \mathbf{S}_{k} \\
& +2 \mathbf{L}_{k}\left\{\lambda_{1}^{-1} \overline{\boldsymbol{\Sigma}}_{k} \mathbf{D}_{k} \mathbf{D}_{k}^{T} \overline{\boldsymbol{\Sigma}}_{k}^{T}\right. \\
& +\breve{\mathbf{\Sigma}}_{k} \circ\left[2\left(a_{1}^{2} \operatorname{tr}\left(\boldsymbol{\Omega}_{k \mid k-1}\right)+a_{2}^{2}\right)\right] \mathbf{I} \\
& \left.+\sum_{i=1}^{r} \mathbf{C}_{i k}^{s} \boldsymbol{\Omega}_{k \mid k-1}\left(\mathbf{C}_{i k}^{s}\right)^{T}+\mathbf{R}_{k}\right\}=0 \\
& \frac{\partial \operatorname{tr}\left(\boldsymbol{\Xi}_{k+1 \mid k+1}\right)}{\partial \mathbf{K}_{k+1}} \\
& =-2\left(\mathbf{I}-\mathbf{K}_{k+1} \overline{\mathbf{\Sigma}}_{k+1} \overline{\mathbf{C}}_{k+1}\right)\left(\boldsymbol{\Xi}_{k+1 \mid k}^{-1}-\lambda_{2} \overline{\mathbf{E}}_{k+1}^{T} \overline{\mathbf{E}}_{k+1}\right)^{-1} \\
& \times \overline{\mathbf{C}}_{k+1}^{T} \bar{\Sigma}_{k+1}
\end{aligned}
$$

$$
\begin{aligned}
+2 \mathbf{K}_{k+1}\{ & \lambda_{2}^{-1} \overline{\boldsymbol{\Sigma}}_{k+1} \overline{\mathbf{D}}_{k+1} \overline{\mathbf{D}}_{k+1}^{T} \overline{\boldsymbol{\Sigma}}_{k+1}^{T} \\
& +\breve{\boldsymbol{\Sigma}}_{k+1} \circ\left[2\left(a_{1}^{2} \operatorname{tr}\left(\boldsymbol{\Delta}_{k+1 \mid k}\right)+a_{2}^{2}\right)\right] \mathbf{I} \\
& \left.+\sum_{i=1}^{r} \mathbf{C}_{i k+1}^{s} \Delta_{k+1 \mid k}\left(\mathbf{C}_{i k+1}^{s}\right)^{T}+\mathbf{R}_{k+1}\right\}=0,
\end{aligned}
$$

where $\boldsymbol{\Omega}_{k \mid k-1}$ and $\boldsymbol{\Delta}_{k+1 \mid k}$ are defined in (30).

Considering (46), the optimized prediction gain $\mathbf{L}_{k}$ and the filtering gain $\mathbf{K}_{k+1}$ can be obtained in (31) and (32). The proof is completed.

For the sake of clarity, the robust recursive filter is summarized as follows.

Step 1. Given $\widehat{\mathbf{x}}_{k \mid k-1}$ and $\boldsymbol{\Xi}_{k \mid k-1}$ and from (31), the prediction gain $\mathbf{L}_{k}$ is computed. Using (5) and (31), the one-step state prediction $\widehat{\mathbf{x}}_{k \mid k-1}$ and the upper bound $\boldsymbol{\Xi}_{k+1 \mid k}$ can be obtained by (6) and (27).

Step 2. The filtering gain $\mathbf{K}_{k+1}$ can be given by (32). The state estimation $\widehat{\mathbf{x}}_{k+1 \mid k+1}$ and the upper bound $\boldsymbol{\Xi}_{k+1 \mid k+1}$ can be given by (7) and (28).

Step 3. Repeat Step 1 to update the one-step state prediction and its upper bound and use Step 2 to obtain the state estimation.

Remark 7. The robust recursive filter problem is removed by using Theorem 6 for nonlinear systems with multiplicative noises, correlated additive noises, and packet losses. Different from the most existing robust filter literature, the robust recursive filter design proposed in this paper is based on the structure including state prediction and state correction in the presence of the correlated additive noises. Note that the phenomenon of correlated additive noises, multiplicative noises, and packet losses arises in the engineering applications. In order to solve this problem, a robust recursive filter is derived to find the upper bound of the prediction error covariance and the filtering error covariance and design the filter parameters to minimize the upper bounds. It is worth mentioning that, though the correction terms in (28) and (32) are similar to the corresponding component in [36], there is a clear difference between the prediction terms in (27) and (31) caused by correlated additive noises and the counterpart in $[36,37]$, which will directly affect the estimation results. This distinguishes our work from the work in $[36,37]$.

\section{Simulation}

To show the effectiveness of the proposed robust recursive filter (RRF), it is compared with the finite-horizon extended Kalman filter (FEKF) in the literature [36] by employing the following examples. 
Example 8. The discretized maneuvering target tracking example in $[36,37]$ is presented including correlated additive noises, multiplicative noises, and packet losses:

$$
\begin{aligned}
\mathbf{x}_{k+1}=\left[\begin{array}{l}
\mathbf{x}_{1, k+1} \\
\mathbf{x}_{2, k+1}
\end{array}\right]= & {\left[\begin{array}{l}
0.8 \mathbf{x}_{1, k}+\mathbf{x}_{1, k} \mathbf{x}_{2, k} \\
1.5 \mathbf{x}_{2, k}-\mathbf{x}_{1, k} \mathbf{x}_{2, k}
\end{array}\right] } \\
& +\left[\begin{array}{ll}
0.06 & 0.08 \\
0.09 & 0.12
\end{array}\right] \boldsymbol{\eta}_{k}\left[\begin{array}{l}
\mathbf{x}_{1, k} \\
\mathbf{x}_{2, k}
\end{array}\right]+\left[\begin{array}{l}
0.01 \\
0.03
\end{array}\right] \mathbf{w}_{k}, \\
\mathbf{y}_{k}= & \mathbf{\Sigma}_{k} \times 7.5 \sin \left(\mathbf{x}_{2, k}\right)+\left[\begin{array}{ll}
0.15 & 0.2
\end{array}\right] \boldsymbol{\xi}_{k}\left[\begin{array}{c}
\mathbf{x}_{1, k} \\
\mathbf{x}_{2, k}
\end{array}\right]+\mathbf{v}_{k},
\end{aligned}
$$

where the state $\mathbf{x}_{k}=\left[\begin{array}{ll}\mathbf{x}_{1, k}^{T} & \mathbf{x}_{2, k}^{T}\end{array}\right]^{T}$ represents the position and velocity of target; $\boldsymbol{\eta}_{k}$ and $\boldsymbol{\xi}_{k}$ are independent zero mean Gaussian white noises with covariance $1 ; \mathbf{w}_{k}$ and $\mathbf{v}_{k}$ are correlated zero mean Gaussian noises with $\mathbf{Q}_{k}=0.05$ and $\mathbf{R}_{k}=0.05$. Let $\mathbf{S}_{k}=0.02$. The mean and covariance of $\boldsymbol{\Sigma}_{k}$ are determined as $\mu_{k}=0.9$ and $\left(\sigma_{k}\right)^{2}=0.065$.

The initial state and covariance are set as $\widehat{\mathbf{x}}_{0 \mid 0}=\left[\begin{array}{ll}1.8 & 0.2\end{array}\right]^{T}$ and $\boldsymbol{\Xi}_{0 \mid 0}=20 \mathbf{I}_{2}$. Let $\varepsilon_{1}=0.4, \varepsilon_{2}=0.35, \lambda_{1}=\lambda_{2}=0.002$, $\mathbf{D}_{k}=\overline{\mathbf{D}}_{k+1}=\left[\begin{array}{ll}0.1 & 0.15\end{array}\right]^{T}, \mathbf{E}_{k}=\overline{\mathbf{E}}_{k+1}=0.01 \mathbf{I}_{2}$, and $\mathbf{B}_{k}=$ $\operatorname{diag}\{0.1,0.2\}$.

To evaluate the performance of the proposed robust recursive filter, the mean square error (MSE) is employed. And it can be expressed as

$$
\mathrm{MSE}=\frac{1}{N} \sum_{k-1}^{N}\left(\mathbf{x}_{k}-\widehat{\mathbf{x}}_{k \mid k}\right)^{2}
$$

where $N$ is the sample number.

Simulation results are shown in Figures 1-4. From Figures 1 and 2, it can be seen that, compared with the FEKF in [36], the proposed robust recursive filter performs better when the model is correlated with additive noises, multiplicative noises, and packet losses. Both true position and velocity are tracked well. This is because the effect of the proposed algorithm can compensate for the correlated noise, while the FEKF cannot. Shown in Figures 3 and 4 are the comparisons of MSE of the estimated states with the corresponding diagonal elements of the estimation error covariance. Obviously, for the proposed algorithm, the MSE of the estimated state is always lower than the upper bound. This confirms the results of Theorem 6. Meanwhile, the MSE of the RRF stays below the MSE of the FEKF, which further illustrates that the proposed algorithm has higher precision than the FEKF in presence of correlated additive noises, multiplicative noises, and packet losses.

Example 9. According to the literature [26], the robust recursive filter is considered to handle the attitude estimation problem with correlated additive noises, multiplicative

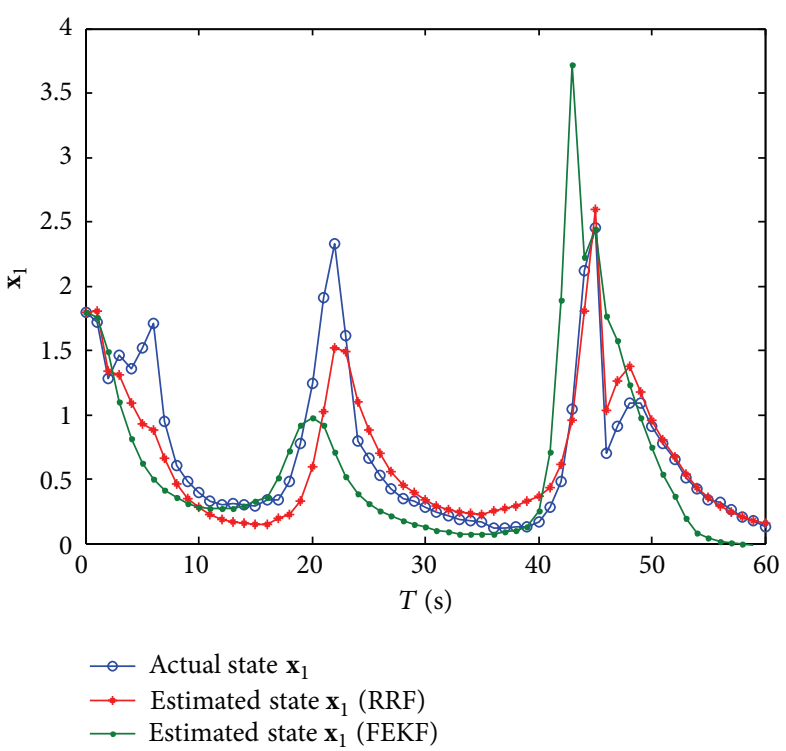

Figure 1: The trajectory of the actual state $\mathbf{x}_{1}$ and its estimate.

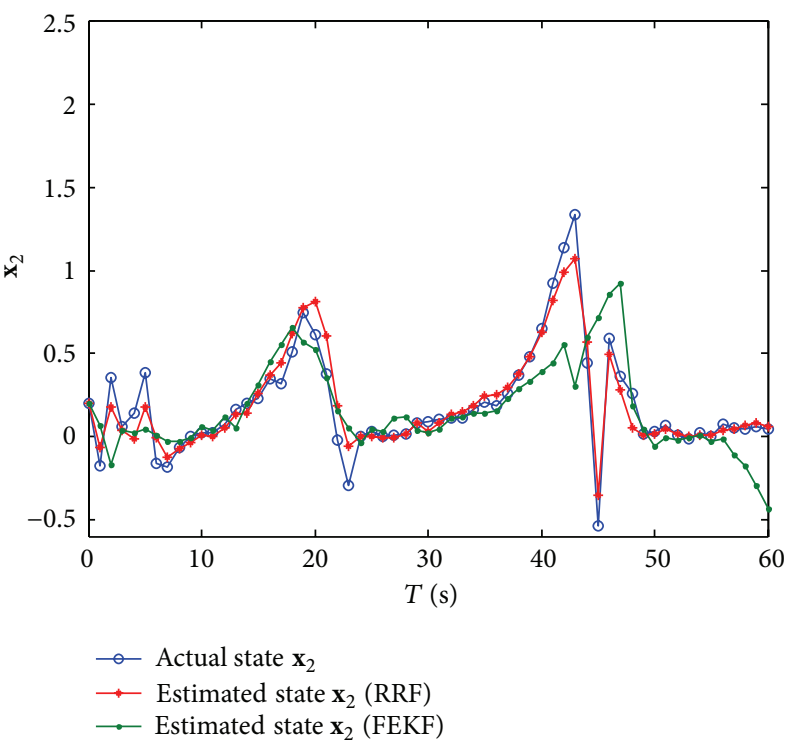

Figure 2: The trajectory of the actual state $\mathbf{x}_{2}$ and its estimate.

noises, and packet losses. The system process models are expressed as follows:

$$
\begin{aligned}
\mathbf{x}_{k+1}=\left[\begin{array}{c}
\mathbf{q}_{k+1} \\
\boldsymbol{\beta}_{k+1}
\end{array}\right]= & {\left[\begin{array}{cc}
\mathbf{I}_{4 \times 4}+\frac{\Delta t}{2} \boldsymbol{\Omega}\left(\widetilde{\boldsymbol{\omega}}_{k}-\boldsymbol{\beta}_{k}\right) & \mathbf{0}_{4 \times 3} \\
\mathbf{0}_{3 \times 4} & \mathbf{I}_{3 \times 3}
\end{array}\right]\left[\begin{array}{c}
\mathbf{q}_{k} \\
\boldsymbol{\beta}_{k}
\end{array}\right] } \\
& +\left[\begin{array}{c}
-\frac{\Delta t}{2} \boldsymbol{\Xi}\left(\mathbf{q}_{k}\right) \\
\mathbf{0}_{3 \times 3}
\end{array}\right] \boldsymbol{\eta}_{v}+\left[\begin{array}{c}
\mathbf{0}_{4 \times 1} \\
\boldsymbol{\eta}_{u}
\end{array}\right] \\
= & f\left(\mathbf{x}_{k}, \widetilde{\boldsymbol{\omega}}_{k}\right)+\sum_{i=1}^{s} \mathbf{A}_{i k} \boldsymbol{\eta}_{i k} \mathbf{x}_{k}+\left[\begin{array}{c}
\mathbf{0}_{4 \times 1} \\
\sigma_{u} \sqrt{\Delta t} \mathbf{I}_{3 \times 1}
\end{array}\right] \mathbf{w}_{k},
\end{aligned}
$$




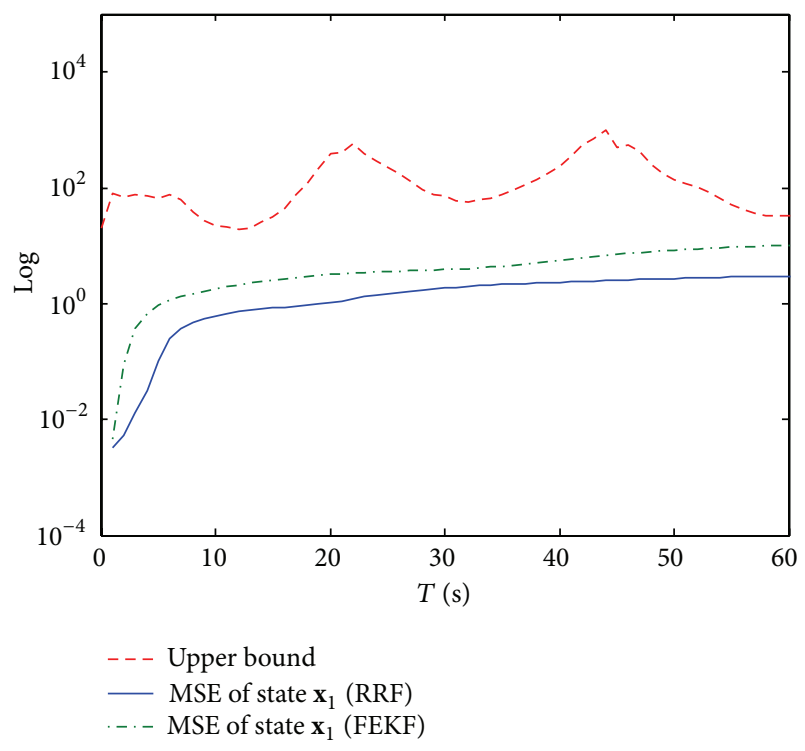

FIgURE 3: MSE of the estimated state $\mathbf{x}_{1}$ and upper bound.

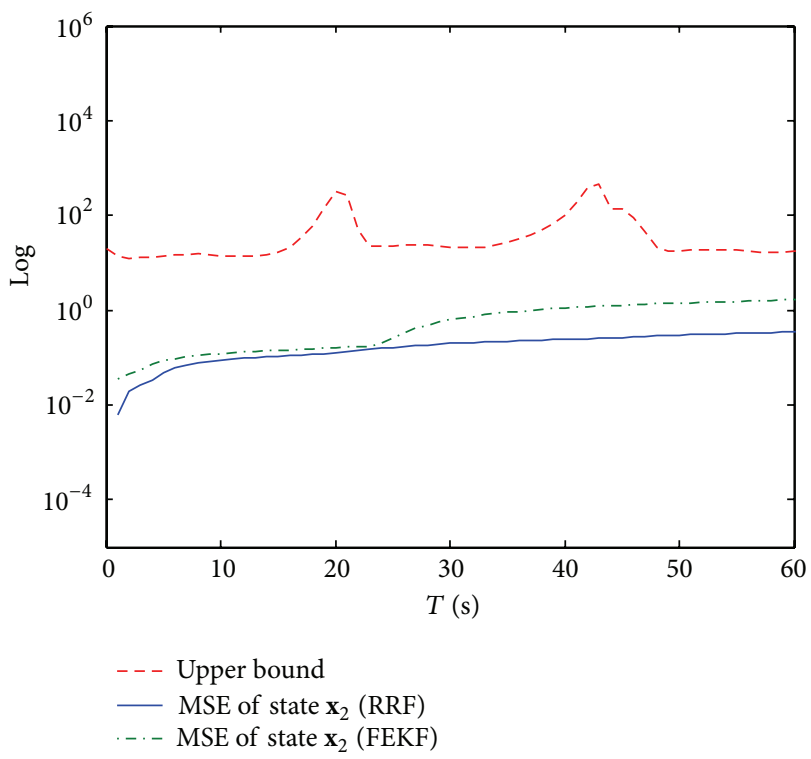

FIGURE 4: MSE of the estimated state $\mathbf{x}_{2}$ and upper bound.

where the state $\mathbf{x}_{k}$ consisted of the quaternion vector $\mathbf{q}_{k}$ and the gyro bias vector $\boldsymbol{\beta}_{k}$; $\widetilde{\boldsymbol{\omega}}_{k}$ is the gyro measured angular rate at time $k ; \Delta t$ is the gyros sampling interval; $\boldsymbol{\eta}_{v}$ is the Gaussian white-noise process with zero mean and covariance $\sigma_{v}^{2} ; \boldsymbol{\eta}_{u}$ is the zero mean Gaussian white-noise process with covariance $\sigma_{u}^{2} \Delta t ; s=3 ; \boldsymbol{\eta}_{i k}$ is the zero mean multiplicative noise with covariance $1 ; \mathbf{w}_{k}$ is the zero mean Gaussian noise with covariance $1 ;[\boldsymbol{\omega} \times]$ is a cross-product matrix defined by

$$
[\boldsymbol{\omega} \times]=\left[\begin{array}{ccc}
0 & -\omega_{3} & \omega_{2} \\
\omega_{3} & 0 & -\omega_{1} \\
-\omega_{2} & \omega_{1} & 0
\end{array}\right], \quad \Omega(\boldsymbol{\omega})=\left[\begin{array}{cc}
-[\boldsymbol{\omega} \times] & \boldsymbol{\omega} \\
-\boldsymbol{\omega}^{T} & 0
\end{array}\right] \text {; }
$$

$\mathbf{A}_{i k}$ are known scaling matrices with appropriate dimension, which can be expressed as

$$
\mathbf{A}_{i k}=-\frac{\Delta t \sigma_{v}}{2}\left[\begin{array}{cc}
\mathbf{A}_{i k}^{1} & \mathbf{0}_{4 \times 3} \\
\mathbf{0}_{3 \times 4} & \mathbf{0}_{3 \times 3}
\end{array}\right],
$$

where

$$
\begin{gathered}
\mathbf{A}_{1 k}^{1}=\left[\begin{array}{cccc}
0 & 0 & 0 & 1 \\
0 & 0 & 1 & 0 \\
0 & -1 & 0 & 0 \\
-1 & 0 & 0 & 0
\end{array}\right], \quad \mathbf{A}_{2 k}^{1}=\left[\begin{array}{cccc}
0 & 0 & -1 & 0 \\
0 & 0 & 0 & 1 \\
1 & 0 & 0 & 0 \\
0 & -1 & 0 & 0
\end{array}\right], \\
\mathbf{A}_{3 k}^{1}=\left[\begin{array}{cccc}
0 & 1 & 0 & 0 \\
-1 & 0 & 0 & 0 \\
0 & 0 & 0 & 1 \\
0 & 0 & -1 & 0
\end{array}\right] .
\end{gathered}
$$

The measurement model can be described as

$$
\mathbf{z}_{k}=\left[\begin{array}{c}
\mathbf{z}_{k}^{1} \\
\mathbf{z}_{k}^{2} \\
\mathbf{z}_{k}^{3}
\end{array}\right]=\boldsymbol{\Sigma}_{k}\left[\begin{array}{l}
\mathbf{A}\left(\mathbf{q}_{k}\right) \overrightarrow{\mathbf{r}}^{1} \\
\mathbf{A}\left(\mathbf{q}_{k}\right) \overrightarrow{\mathbf{r}}^{2} \\
\mathbf{A}\left(\mathbf{q}_{k}\right) \overrightarrow{\mathbf{r}}^{3}
\end{array}\right]+\left[\begin{array}{c}
\mathbf{v}_{k}^{1} \\
\mathbf{v}_{k}^{2} \\
\mathbf{v}_{k}^{3}
\end{array}\right]=\boldsymbol{\Sigma}_{k} h\left(\mathbf{x}_{k}\right)+\sigma_{s} \mathbf{I}_{9 \times 1} \mathbf{v}_{k},
$$

where $\mathbf{z}_{k}^{i}(i=1,2,3)$ is the measurement vector; $\mathbf{A}\left(\mathbf{q}_{k}\right)$ is the real attitude matrix at time $k ; \overrightarrow{\mathbf{r}}^{i}(i=1,2,3)$ is the reference vector of the star sensors; $i$ is the number of star sensors. $\mathbf{v}_{k}^{i}$ is a zero mean Gaussian white noise with covariance matrix $\sigma_{s}^{2} \mathbf{I}_{3 \times 3} ; \mathbf{v}_{k}$ is the zero mean Gaussian noise with covariance 1 ; if $\mathbf{q}=\left[q_{1}, q_{2}, q_{3}, q_{4}\right]^{T}=\left[\boldsymbol{\rho}^{T}, q_{4}\right]^{T}$, the attitude matrix can be written as

$$
\mathbf{A}(\mathbf{q})=\left(q_{4}^{2}-\boldsymbol{\rho}^{T} \boldsymbol{\rho}\right) \mathbf{I}_{3 \times 3}+2 \boldsymbol{\rho} \boldsymbol{\rho}^{T}-2 q_{4}[\boldsymbol{\rho} \times] .
$$

The simulation conditions are set as follows: the gyro sampling interval is $\Delta t=0.25 \mathrm{~s}$; the standard deviation of gyros' measurement noise is $\sigma_{v}=1.45444 \times 10^{-6} \mathrm{rad} / \mathrm{s}^{1 / 2}$; the standard deviation of gyros' drift noise is $\sigma_{u}=1.3036 \times$ $10^{-9} \mathrm{rad} / \mathrm{s}^{3 / 2} ; \mathbf{w}_{k}$ and $\mathbf{v}_{k}$ are correlated zero mean Gaussian noises with $\mathbf{Q}_{k}=1$ and $\mathbf{R}_{k}=1$; let $\mathbf{S}_{k}=0.5$; the standard deviation of star sensors' measurement noise is all $\sigma_{s}=18^{\prime \prime}$; due to using three star sensors, the reference vectors of the star sensors are $\overrightarrow{\mathbf{r}}^{1}=\left[\begin{array}{lll}1 & 0 & 0\end{array}\right]^{T}, \overrightarrow{\mathbf{r}}^{2}=\left[\begin{array}{lll}0 & 1 & 0\end{array}\right]^{T}$, and $\overrightarrow{\mathbf{r}}^{3}=$ $\left[\begin{array}{lll}0 & 0 & 1\end{array}\right]^{T}$; because of the high precision of the star sensors, the estimation error is rather small in the attitude estimation system. Therefore, set $\mathbf{B}_{k}=\mathbf{0}, \mathbf{D}_{k}=\overline{\mathbf{D}}_{k+1}=\mathbf{0}$. The random variables $\mu_{k}^{i}(\mathrm{i}=1,2, \ldots, 9)$ satisfy the Bernoulli distribution with $\overline{\boldsymbol{\Gamma}}_{k}=\operatorname{diag}\{0.8,0.8,0.8,0.9,0.9,0.9,0.95,0.95,0.95\}$; let $\varepsilon_{1}=\varepsilon_{2}=0.1$ and $\lambda_{1}=\lambda_{2}=0.0001 ;$ let $\mathbf{C}_{i k+1}=\mathbf{0}, a_{1}=1$, and $a_{2}=0.05$.

The simulated results are shown in Figures 5 and 6 . In Figure 5, blue lines represent the quaternion estimation errors of the RRF, green dashed lines represent the quaternion estimation errors of the FEKF, and red dashed lines show the corresponding diagonal elements of the error covariance of the RRF. It can be seen clearly that the estimation errors of the quaternion vector part of the RRF are generally within 


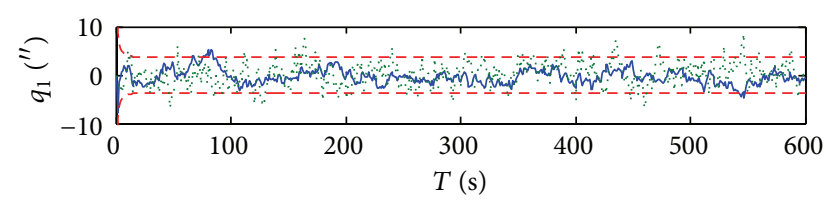

(a)

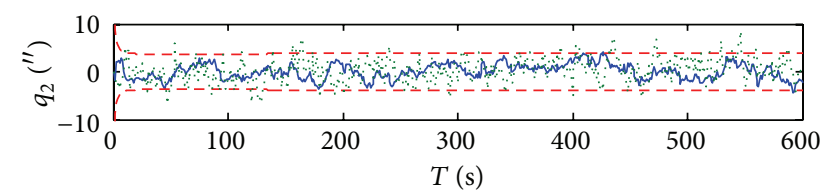

(b)

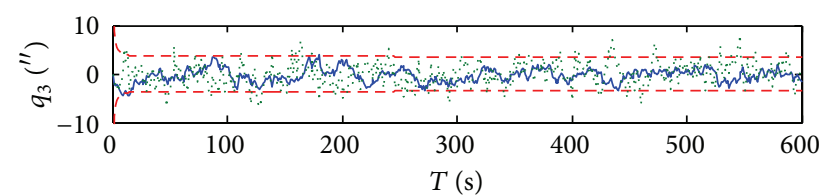

(c)

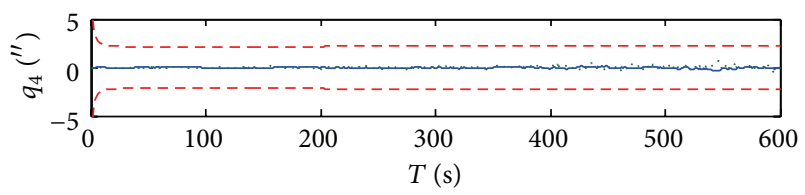

(d)

FIGURE 5: The quaternion estimation errors of the proposed filter.

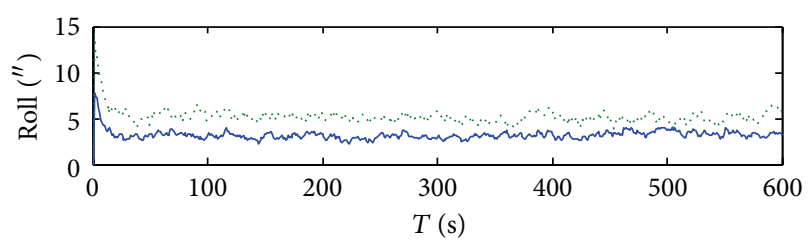

(a)

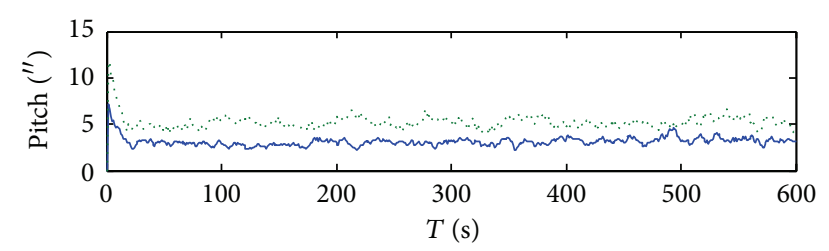

(b)

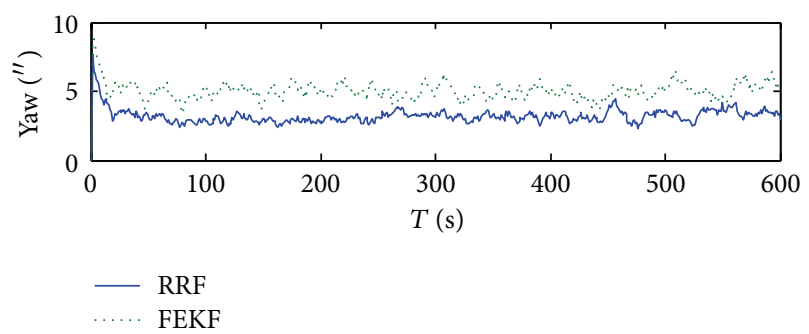

(c)

FIGURE 6: RMSE of attitude angles in the proposed filter. the boundaries of the computed covariance, which indicates that the proposed filter can control correlated additive noises, multiplicative noises, and packet losses. Besides, the quaternion estimation errors of the RRF are smaller than the quaternion estimation errors of the FEKF. These show that the RRF performs better than the FEKF. Furthermore, since it is very important for attitude estimation to get the angle information, the estimated quaternion needs to be converted as the form of Euler angles. In Figure 6, root mean square error (RMSE) is employed to express the quality of the Euler angle estimation. For the RRF, the means of RMSE of the attitude angles are $3.199^{\prime \prime}, 3.125^{\prime \prime}$, and $3.195^{\prime \prime}$, respectively, which are lower than the REKF obviously. The reason why our method has such advantages is that the effects of arising multiplicative noises, packet losses, and correlated additive noises are all compensated for without loss of generality.

\section{Conclusion}

Due to the fact that existing robust filtering algorithms are difficult in dealing with correlated additive noises, a robust recursive filter is developed in this paper for nonlinear systems with consideration of correlated additive noises, multiplicative noises, and packet losses. The proposed algorithm is designed to minimize the upper bound on the prediction covariance and the filtering covariance. Simulated results demonstrate that the proposed filter provides effective performance for controlling correlated additive noises, multiplicative noises, and packet losses.

\section{Conflict of Interests}

The authors declare that there is no conflict of interests regarding the publication of this paper.

\section{References}

[1] D. Choukroun, I. Y. Bar-Itzhack, and Y. Oshman, "Novel quaternion Kalman filter," IEEE Transactions on Aerospace and Electronic Systems, vol. 42, no. 1, pp. 174-190, 2006.

[2] D. H. Dini, C. Jahanchahi, and D. P. Mandic, "Kalman filtering for widely linear complex and quaternion valued bearings only tracking," IET Signal Processing, vol. 6, no. 5, pp. 435-445, 2012.

[3] F. Gustafsson and G. Hendeby, "Some relations between extended and unscented Kalman filters," IEEE Transactions on Signal Processing, vol. 60, no. 2, pp. 545-555, 2012.

[4] J. L. Crassidis, "Sigma-point Kalman filtering for integrated GPS and inertial navigation," IEEE Transactions on Aerospace and Electronic Systems, vol. 42, no. 2, pp. 750-756, 2006.

[5] C. K. Ahn and P. S. Kim, "New peak-to-peak state-space realization of direct form interfered digital filters free of overflow limit cycles," International Journal of Innovative Computing Information and Control, vol. 9, no. 8, pp. 3285-3291, 2013.

[6] H. H. Dam, "Variable fractional delay filter with sub-expression coefficients," International Journal of Innovative Computing, Information and Control, vol. 9, no. 7, pp. 2995-3003, 2013.

[7] T. Miyata and N. Aikawa, "A design of FIR filters with variable notches considering reduction method of polynomial coefficients for real-time signal processing," International Journal of 
Innovative Computing, Information and Control, vol. 9, no. 9, pp. 3527-3536, 2013.

[8] H. Q. Wang, B. Chen, and C. Lin, "Adaptive neural tracking control for a class of stochastic nonlinear systems with unknown dead-zone," International Journal of Innovative Computing Information and Control, vol. 9, no. 8, pp. 3257-3269, 2013.

[9] M. Arounassalame, "Analysis of nonlinear electrical circuits using bernstein polynomials," International Journal of Automation and Computing, vol. 9, no. 1, pp. 81-86, 2012.

[10] R. E. Kalman, "A new approach to linear filtering and prediction problems," Transactions of the ASME-Journal of Basic Engineering, vol. 82, pp. 35-45, 1960.

[11] D. Del Gobbo, M. Napolitano, P. Famouri, and M. Innocenti, "Experimental application of extended Kalman filtering for sensor validation," IEEE Transactions on Control Systems Technology, vol. 9, no. 2, pp. 376-380, 2001.

[12] S. Julier, J. Uhlmann, and H. F. Durrant-Whyte, "A new method for the nonlinear transformation of means and covariances in filters and estimators," IEEE Transactions on Automatic Control, vol. 45, no. 3, pp. 477-482, 2000.

[13] P. Li, J. Lam, and Z. Shu, " $H_{\infty}$ positive filtering for positive linear discrete-time systems: an augmentation approach," IEEE Transactions on Automatic Control, vol. 55, no. 10, pp. 23372342, 2010.

[14] Z. Wang, B. Shen, and X. Liu, " $H_{\infty}$ filtering with randomly occurring sensor saturations and missing measurements," Automatica, vol. 48, no. 3, pp. 556-562, 2012.

[15] A. Hmamed, C. E. Kasri, E. H. Tissir, T. Alvarez, and F. Tadeo, "Robust filtering for uncertain 2-D continuous systems with delays," International Journal of Innovative Computing, Information and Control, vol. 9, no. 5, pp. 2167-2183, 2013.

[16] X. F. Hong, Y. Z. Wang, and H. T. Li, "Robust $H_{\infty}$ filter design for time-delay systems with saturation," International Journal of Automation and Computing, vol. 10, no. 4, pp. 368-374, 2013.

[17] P. N. Pathirana, S. W. Ekanayake, and A. V. Savkin, "Fusion based 3D tracking of mobile transmitters via robust set-valued state estimation with RSS measurements," IEEE Communications Letters, vol. 15, no. 5, pp. 554-556, 2011.

[18] A. N. Bishop, A. V. Savkin, and P. N. Pathirana, "Vision-based target tracking and surveillance with robust set-valued state estimation," IEEE Signal Processing Letters, vol. 17, no. 3, pp. 289292, 2010.

[19] E. N. Gonçalves, R. M. Palhares, and R. H. C. Takahashi, " $H_{2} / H_{\infty}$ filter design for systems with polytope-bounded uncertainty," IEEE Transactions on Signal Processing, vol. 54, no. 9, pp. 3620-3626, 2006.

[20] L. Xie, L. Lu, D. Zhang, and H. Zhang, "Improved robust $H_{2}$ and $H_{\infty}$ filtering for uncertain discrete-time systems," Automatica, vol. 40, no. 5, pp. 873-880, 2004.

[21] A. G. Kallapur, I. R. Petersen, and S. G. Anavatti, "A discretetime robust extended kalman filter for uncertain systems with sum quadratic constraints," IEEE Transactions on Automatic Control, vol. 54, no. 4, pp. 850-854, 2009.

[22] K. Xiong, L. Liu, and Y. Liu, "Non-linear robust filter design for satellite attitude determination," IET Control Theory and Applications, vol. 4, no. 7, pp. 1222-1234, 2010.

[23] F. Wang and V. Balakrishnan, "Robust Kalman filters for linear time-varying systems with stochastic parametric uncertainties," IEEE Transactions on Signal Processing, vol. 50, no. 4, pp. 803813, 2002.
[24] F. Wang and V. Balakrishnan, "Robust steady-state filtering for systems with deterministic and stochastic uncertainties," IEEE Transactions on Signal Processing, vol. 51, no. 10, pp. 2550-2558, 2003.

[25] F. Yang, Z. Wang, and Y. S. Hung, "Robust Kalman filtering for discrete time-varying uncertain systems with multiplicative noises," IEEE Transactions on Automatic Control, vol. 47, no. 7, pp. 1179-1183, 2002.

[26] X. Kai, L. Liangdong, and L. Yiwu, "Robust extended Kalman filtering for nonlinear systems with multiplicative noises," Optimal Control Applications and Methods, vol. 32, no. 1, pp. 47-63, 2011.

[27] X. Su, L. Wu, and P. Shi, "Sensor networks with random link failures: distributed filtering for T-S fuzzy systems," IEEE Transactions on Industrial Informatics, vol. 9, no. 3, pp. 17391750, 2013.

[28] X. Su, P. Shi, L. Wu, and S. K. Nguang, "Induced $l_{2}$ filtering of fuzzy stochastic systems with time-varying delays," IEEE Transactions on Cybernetics, vol. 43, no. 4, pp. 1251-1264, 2013.

[29] L. Wu and D. W. C. Ho, "Fuzzy filter design for Itô stochastic systems with application to sensor fault detection," IEEE Transactions on Fuzzy Systems, vol. 17, no. 1, pp. 233-242, 2009.

[30] R. Yang, P. Shi, and G.-P. Liu, "Filtering for discrete-time networked nonlinear systems with mixed random delays and packet dropouts," IEEE Transactions on Automatic Control, vol. 56, no. 11, pp. 2655-2660, 2011.

[31] R. N. Yang, G. P. Liu, P. Shi, C. Thomas, and M. V. Basin, "Predictive output feedback control for networked control systems," IEEE Transactions on Industrial Electronics, vol. 61, no. 1, pp. 512-520, 2014.

[32] S. Sun, L. Xie, W. Xiao, and Y. C. Soh, "Optimal linear estimation for systems with multiple packet dropouts," Automatica, vol. 44, no. 5, pp. 1333-1342, 2008.

[33] S. Kluge, K. Reif, and M. Brokate, "Stochastic stability of the extended kalman filter with intermittent observations," IEEE Transactions on Automatic Control, vol. 55, no. 2, pp. 514-518, 2010.

[34] M. Basin, P. Shi, and D. Calderon-Alvarez, "Approximate finitedimensional filtering for polynomial states over polynomial observations," International Journal of Control, vol. 83, no. 4, pp. 724-730, 2010.

[35] B. Wang, G. Guo, and W. Yue, "Variance-constrained robust estimation for uncertain systems with multiple packet dropouts," Optimal Control Applications and Methods, vol. 34, no. 1, pp. 53-68, 2013.

[36] J. Hu, Z. D. Wang, H. J. Gao, and L. K. Stergioulas, "Extended Kalman filtering with stochastic nonlinearities and multiple missing measurements," Automatica, vol. 48, pp. 2007-2015, 2012.

[37] J. Hu, Z. D. Wang, B. Shen, and H. J. Gao, "Quantised recursive filtering for a class of nonlinear systems with multiplicative noises and missing measurements," International Journal of Control, vol. 86, no. 4, pp. 650-663, 2013.

[38] X. Jiahe, G. M. Dimirovski, J. Yuanwei, and S. Chao, "UKF design and stability for nonlinear stochastic systems with correlated noises," in Proceedings of the 46th IEEE Conference on Decision and Control (CDC '07), pp. 6226-6231, December 2007.

[39] X. X. Wang, Y. Liang, Q. Pan, and F. Yang, "A Gaussian approximation recursive filter for nonlinear systems with correlated noises," Automatica, vol. 48, no. 9, pp. 2290-2297, 2012. 
[40] R. A. Horn and C. R. Johnson, Topic in Matrix Analysis, Cambridge University Press, New York, NY, USA, 1991.

[41] L. Xie, Y. C. Soh, and C. E. de Souza, "Robust Kalman filtering for uncertain discrete-time systems," IEEE Transactions on Automatic Control, vol. 39, no. 6, pp. 1310-1314, 1994.

[42] Y. Theodor and U. Shaked, "Robust discrete-time minimumvariance filtering," IEEE Transactions on Signal Processing, vol. 44, no. 2, pp. 181-189, 1996. 


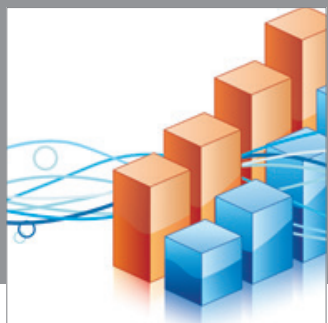

Advances in

Operations Research

mansans

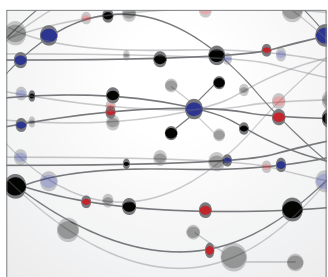

The Scientific World Journal
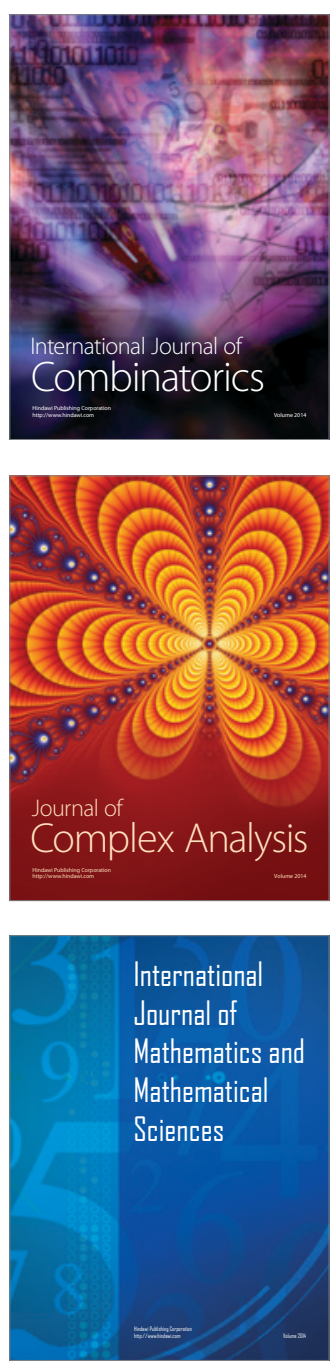
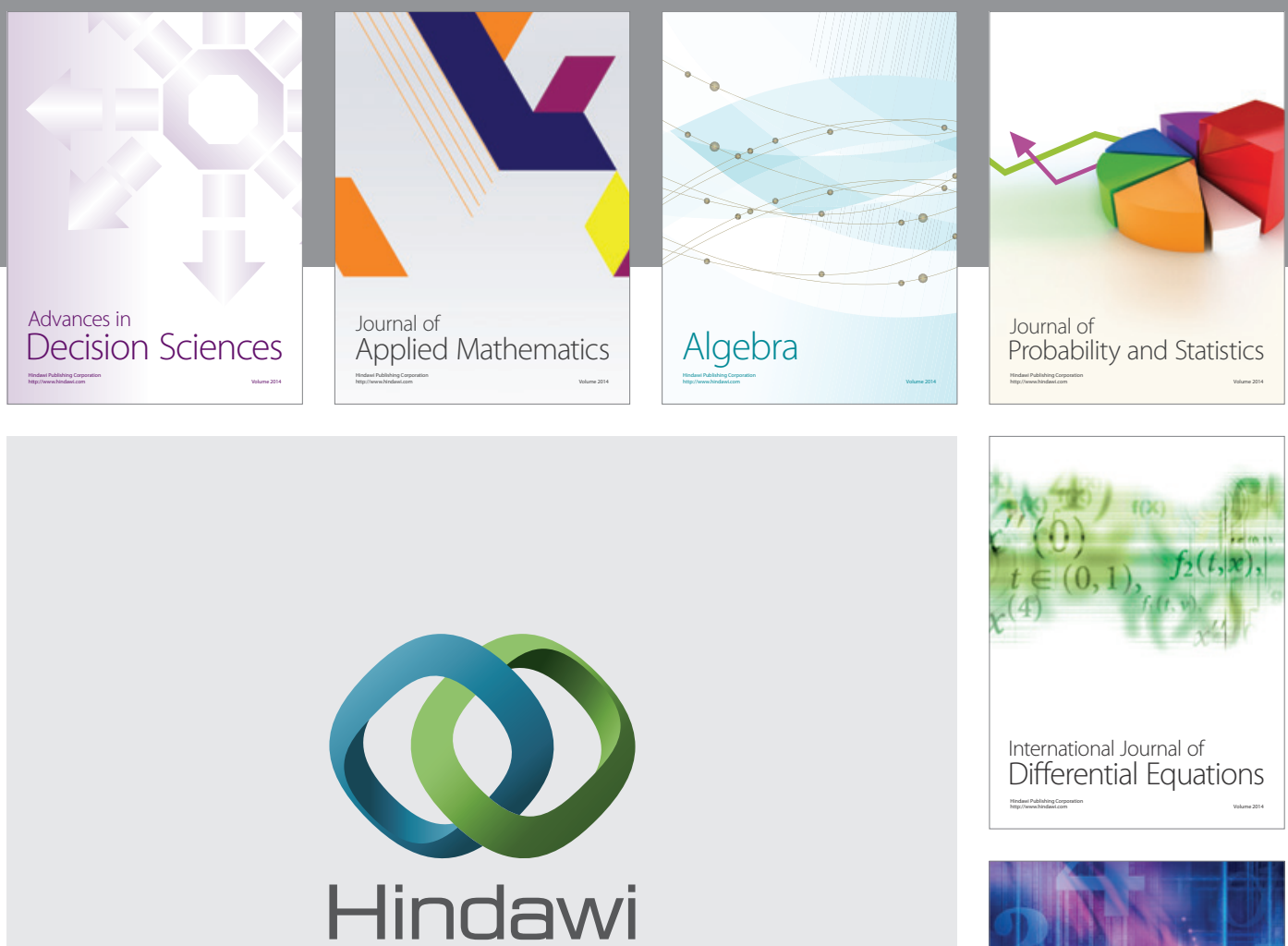

Submit your manuscripts at http://www.hindawi.com
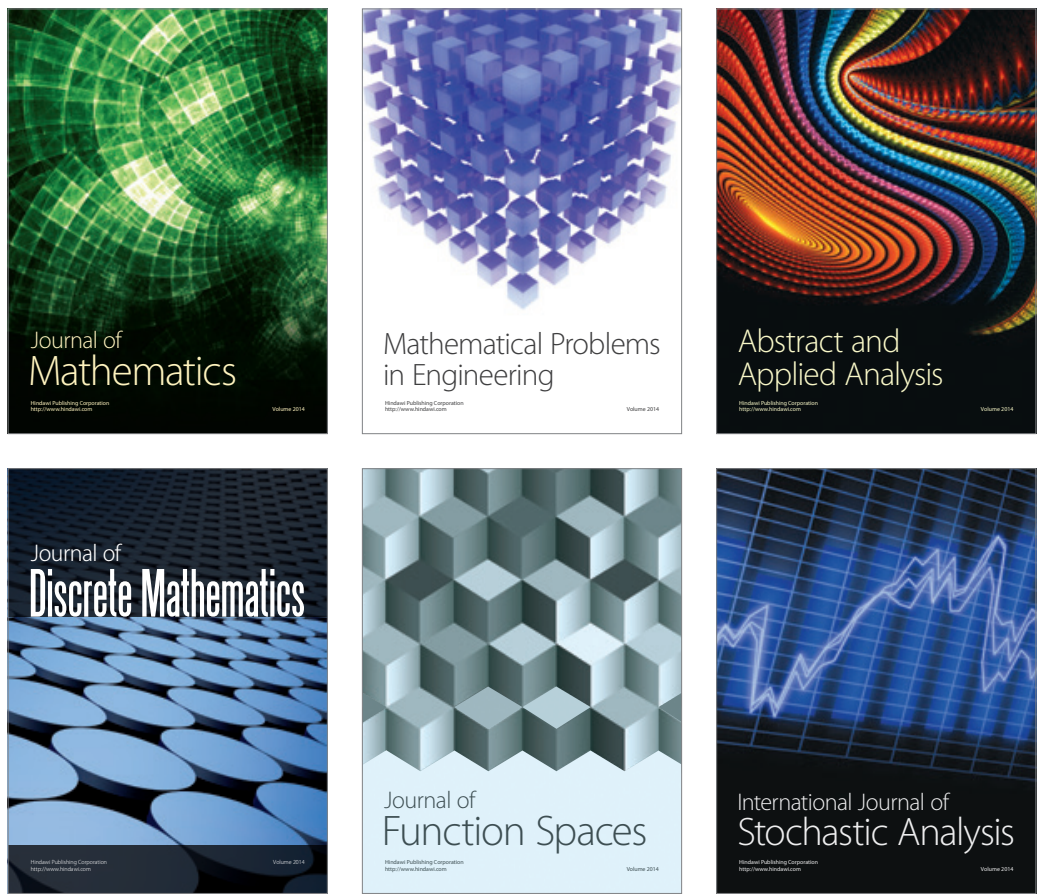

Journal of

Function Spaces

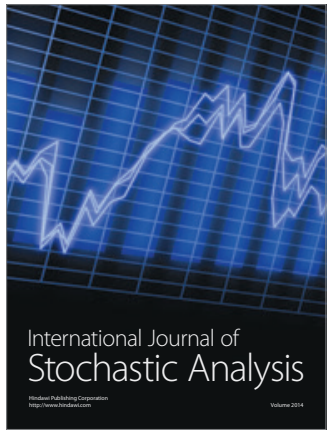

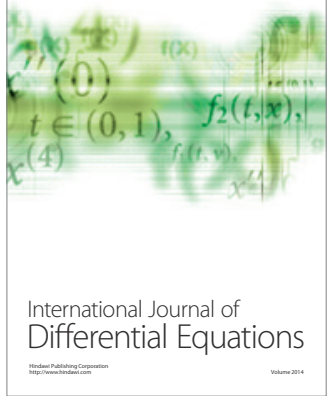
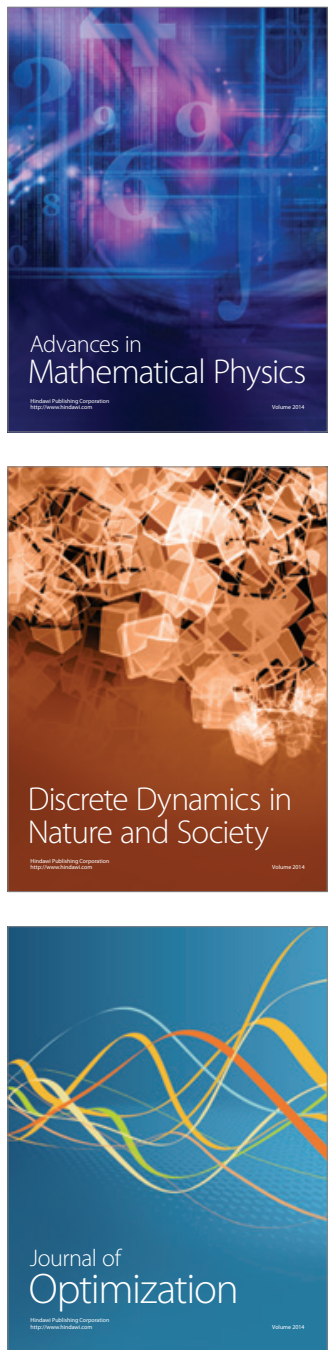